OPEN ACCESS

Edited by: Long-Jun Wu, Mayo Clinic, United States

Reviewed by:

Wei Qiu,

Third Affiliated Hospital of Sun Yat-sen

University, China

Pingting Liu,

Stanford University, United States

*Correspondence:

Ying Xu

yingxu825@126.com

${ }^{+}$These authors have contributed equally to this work

Specialty section: This article was submitted to Multiple Sclerosis and Neuroimmunology, a section of the journal

Frontiers in Immunology

Received: 10 August 2020 Accepted: 10 November 2020 Published: 09 December 2020

Citation:

Yu Y, Wu D-M, Li J, Deng S-H, Liu T, Zhang $T$, He $M$, Zhao $Y$ - $Y$ and $X u Y$ (2020) Bixin Attenuates Experimental Autoimmune Encephalomyelitis

by Suppressing TXNIP/NLRP3 Inflammasome Activity and Activating NRF2 Signaling.

Front. Immunol. 11:593368. doi: 10.3389/fimmu.2020.593368

\section{Bixin Attenuates Experimental Autoimmune Encephalomyelitis by Suppressing TXNIP/NLRP3 Inflammasome Activity and Activating NRF2 Signaling}

Ye $\mathrm{Yu}^{+}$, Dong-Ming $\mathrm{Wu}^{+}$, Jing Li, Shi-Hua Deng, Teng Liu, Ting Zhang, Miao He, Yang-Yang Zhao and Ying $\mathrm{Xu}$ *

\section{Clinical Medical College, The First Affiliated Hospital, Collaborative Innovation Center of Sichuan for Elderly Care and Health} of Chengdu Medical College, Chengdu, China

Multiple sclerosis (MS), an autoimmune and degenerative disease, is characterized by demyelination and chronic neuroinflammation. Bixin is a carotenoid isolated from the seeds of Bixa orellana that exhibits various potent pharmacological activities, including antioxidant, anti-inflammatory, and anti-tumor properties. However, the effects of bixin on MS have not yet been examined. To evaluate the effects and underlying molecular mechanisms of bixin on MS, experimental autoimmune encephalomyelitis (EAE) was established in C57BL/6 mice, which were treated via intragastric administration of bixin solutions. To evaluate the molecular mechanisms of bixin, quantitative reversetranscription PCR, western blot, immunohistochemistry, flow cytometry, and enzymelinked immunosorbent assay analyses were performed. We found that bixin significantly improved the symptoms and pathology in EAE mice, reduced the release of inflammatory cytokines TNF- $\alpha, \mathrm{IL}-6, \mathrm{IL}-8, \mathrm{IL}-17$, and IFN- $\gamma$, and increased the expression of the antiinflammatory cytokine IL-10. And bixin reduced the proportion of Th1 and Th17 cells in the spleen and CNS, and suppressed microglia aggregation, and TXNIP/NLRP3 inflammasome activity by scavenging excessive reactive oxygen species (ROS) in EAE mice. Furthermore, bixin inhibited inflammation and oxidative stress via activating nuclear factor erythroid 2-related factor 2 (NRF2), and its downstream genes in EAE mice, meanwhile, these effects were suppressed upon treatment with an NRF2 inhibitor, ML385. Bixin prevented neuroinflammation and demyelination in EAE mice primarily by scavenging ROS through activation of the NRF2 signaling pathway. Taken together, our results indicate that bixin is a promising therapeutic candidate for treatment of MS.

Keywords: bixin, experimental autoimmune encephalomyelitis, thioredoxin-interacting protein, NLRP3, nuclear factor erythroid 2-related factor 2, reactive oxygen species 


\section{INTRODUCTION}

Multiple sclerosis (MS) is a chronic autoimmune and degenerative disease of the central nervous system (CNS), and the most frequent cause of neurological disability in young adults (1). The pathological changes in patients with MS are multifaceted, including multiple demyelinating plaques, and are accompanied by glial activation and axonal damage (2-6). Although many studies have proposed possible molecular mechanisms and therapeutic strategies for MS, its pathology is largely unknown, involving apoptosis, oxidative stress, and inflammation. As such, effective treatments remain elusive $(7,8)$.

A recent study has proven that the NLRP3 inflammasome plays a pivotal role in the pathogenesis of neuroinflammation and demyelination in EAE (5). The thioredoxin-interacting protein (TXNIP)-NLRP3 inflammasome is a macromolecular polyprotein complex, composed of TXNIP, NLRP3, the junction protein ASC, as well as the effector protein caspase-1. Excessive reactive oxygen species (ROS) accumulation, induced by high glucose levels, increases TXNIP expression. Accumulated TXNIP, in turn, activates the NLRP 3 inflammasome, consequently inducing the secretion of IL-1 $\beta$ and IL-18 as part of the inflammatory response (9-12). Furthermore, activated NLRP3 inflammasomes induce the expression of gasdermin D (GSDMD), which leads to the release of numerous inflammatory cytokines, exacerbating the detrimental inflammatory response (13). Hence, blocking TXNIP-NLRP3 activity is expected to alleviate neuroinflammation and demyelination.

Bixin is a natural chemo-preventive carotenoid isolated from the seeds of Bixa orellana that is capable of crossing the bloodbrain barrier (14-16). Bixin is a lineal apocarotenoid of 25 carbon atoms with nine double bonds and has demonstrated various potent pharmacological activities, including antioxidant, anti-inflammatory, and anti-tumor properties $(17,18)$. Previous studies have demonstrated that bixin can ameliorate high-fatdiet-induced cardiac injury and PM2.5 particle-induced lung injury through suppressing inflammation and oxidative stress $(19,20)$. The present study evaluated the effects of bixin on neuroinflammation and demyelination in a mouse model of autoimmune encephalomyelitis, with the aim of assessing whether bixin could serve as an effective therapeutic compound for MS.

\section{MATERIALS AND METHODS}

\section{Antibodies}

The primary antibodies used for western blot assay and immunohistochemical analysis were as follows: anti-GAPDH (10494-1-AP), anti-CD3 (17617-1-AP), anti-MBP (10458-1AP), anti-IBA1 (10904-1-AP), anti-CD68 (28058-1-AP), and horseradish peroxidase (HRP)-conjugated secondary antibodies (SA00001-2) used for western blot analysis were purchased from Proteintech (Wuhan, China); anti-Catalase (CAT, 14097), antiSOD2 (13141S) (Cell Signaling Technology, Danvers, MA, USA); anti-3-Nitrotyrosine (3-NT, AB5411, Millipore); anti-NAD(P)H dehydrogenase quinone 1 (NQO-1, ab34173), anti-NRF2 (ab31163), anti-TXNIP (ab232330), anti-NLRP3 (ab4207), anti-Caspase-1 (ab179515), anti-IL-18 (ab68435), anti-IL-1 $\beta$ (ab2105), and anti-ASC (ab127537) were purchased from Abcam (Cambridge, UK);. Cy3 goat anti-rabbit IgG $(\mathrm{H}+\mathrm{L})$ (A0516) and Alexa Fluor 488 goat anti-mouse IgG $(\mathrm{H}+\mathrm{L})$ (A0428) used for immunofluorescence analysis were purchased from Beyotime (Shanghai, China). The SPlink detection kits used for immunohistochemical analysis were purchased from ZSGBBIO Technology (SP-9000) (Beijing, China). The antibodies used for flow cytometry analysis were as follows: PE-CYANINE antimouse IL-17 (25-7177-80), PE-CYANINE anti-mouse IFN- $\gamma$ (25-7311-82), FITC anti-mouse CD4 (11-0041-82) (eBioscience, San Diego, CA, USA).

\section{Establishment of the EAE Model}

Seven-week-old female C57BL/6 mice were purchased from Chengdu Dashuo Experimental Animal Company (Chengdu, China). All animal procedures were approved by the Animal Policy and Welfare Committee of Chengdu Medical College (CDYXY-2019036). All mice were housed in a specific pathogen-free facility with a 12 -h light/dark cycle and provided with regular food and water during the acclimatization period.

The EAE model was established as previously described (21). Myelin oligodendrocyte glycoprotein ${ }_{35-55}\left(\mathrm{MOG}_{35-55}\right)$ peptide (Hook Labs, USA) $(200 \mu \mathrm{g})$ was dissolved in $100 \mu \mathrm{l}$ of phosphate-buffered saline (PBS) and emulsified with $100 \mu \mathrm{l}$ of complete Freund's adjuvant (CFA; Chondrex, USA) supplemented with $400 \mu \mathrm{g}$ Mycobacterium tuberculosis $\mathrm{H} 37 \mathrm{Ra}$ (Difco, BD Biosciences, USA). Next, the above emulsions were subcutaneously injected into the mice (Day 1). Pertussis toxin (PTX; List Biological Labs, Campbell, CA, USA) (300 ng) was intraperitoneally administered on the first and third days post-immunization.

\section{Bixin Treatment}

Animals were randomly divided into the following five groups: healthy control (PBS, $\mathrm{n}=5), \mathrm{EAE}(\mathrm{n}=5), \mathrm{EAE}+$ bixin $(50 \mathrm{mg} /$ $\mathrm{kg}$, once daily; $\mathrm{n}=5), \mathrm{EAE}+$ bixin $(100 \mathrm{mg} / \mathrm{kg}$, once daily; $\mathrm{n}=5)$, and $\mathrm{EAE}+$ bixin $(200 \mathrm{mg} / \mathrm{kg}$, once daily; $\mathrm{n}=5)$. Bixin $(6983-79$ 5, MedChemExpress, Shanghai, China) was dissolved in dimethyl sulfoxide (DMSO) at $2 \mathrm{~g} / \mathrm{ml}$ and then diluted with PBS. Starting at 12 days post-immunization, mice in the EAE + bixin group were administered intragastrically with bixin solutions $(50,100$, or $200 \mathrm{mg} / \mathrm{kg} /$ day) for 18 days. On day 30 post-immunization, the mice from each group were euthanized, the spinal cords, brains, spleens, and peripheral blood were collected and used for further experiments.

\section{NRF2 Inhibitor Treatment}

The NRF2 inhibitor ML385 (MCE, Shanghai, China) was dissolved in DMSO at $300 \mathrm{mg} / \mathrm{ml}$ and diluted with PBS (22). Mice were randomly assigned to the following eight groups: healthy control (PBS, $\mathrm{n}=5), \mathrm{EAE}(\mathrm{n}=5), \mathrm{EAE}+$ bixin $(100 \mathrm{mg} /$ $\mathrm{kg}$, once daily; $\mathrm{n}=5)$, bixin $(100 \mathrm{mg} / \mathrm{kg}$, once daily; $\mathrm{n}=5)$, ML385 ( $=5)$, ML385 + EAE ( $=5)$, ML385 + EAE + bixin $(100 \mathrm{mg} / \mathrm{kg}$, once daily; $\mathrm{n}=5)$, and ML385 + bixin $(100 \mathrm{mg} / \mathrm{kg}$, once daily; $\mathrm{n}=5)$. ML385 (30 mg/kg) pre-treatment 
was intraperitoneally administered $1 \mathrm{~h}$ before intragastric administration of bixin.

\section{Bodyweight and Behavioral Assessments}

Clinical behavior scores of each group were evaluated as per the following criteria: 0 , without symptoms; 1 , loss of tail tension; 2 , flaccid hind limb; 3, moderate hind limb paralysis; 4, paralysis of both hind limbs and forelimbs, or accompanied with urinary and fecal disorders; and 5, near-death state (2). The body weight was recorded daily.

\section{Hematoxylin and Eosin (H\&E) and Luxury Fast Blue (LFB) Staining}

Mice were anesthetized using $0.6 \%$ pentobarbital sodium ( $40 \mathrm{mg} /$ $\mathrm{kg}$ ), and the lumbar enlargements of spinal cords and the right hemispheres of brains tissues were collected and fixed with $4 \%$ paraformaldehyde (in PBS) for $24 \mathrm{~h}$ at room temperature, dehydrated with an ethanol gradient and cleared with xylene, subsequently embedded in paraffin, and then cut into 5$\mu \mathrm{m}$ sections.

To evaluate the degree of inflammatory cell infiltration, brain sections and spinal cords were stained using an $\mathrm{H} \& \mathrm{E}$ staining kit (Beyotime Biotechnology, Shanghai, China). The sections were dewaxed and dehydrated, subsequently washed with PBS, and then stained with hematoxylin and eosin for $2 \mathrm{~min}$, respectively. The inflammatory infiltration was evaluated as previously reported (23), 0 = no inflammatory cells; $1=$ a few scattered inflammatory cells; 2 = organization of inflammatory infiltrates around blood vessels; 3 = extensive perivascular cuffing with extension into parenchyma.

The spinal cords were stained with LFB staining solution (Solarbio, Beijing, China), to evaluate changes in demyelination. The sections were stained with modified page staining solution and page peach red dye solution, respectively, after being dewaxed and dehydrated with an ethanol gradient. The demyelination scores were evaluated as previously reported (23), 0 = none; 1 = rare foci; 2 = a few areas of demyelination; and 3 = large areas of demyelination. The images were randomly captured at 20× magnification (XI 71 Olympus, Tokyo, Japan).

\section{Bielschowsky Staining}

To determine the degree of axon degeneration, the spinal cords sections were stained using a Bielschowsky staining kit (Bioss, Beijing, China). The sections were dewaxed and dehydrated, subsequently washed with distilled water, and then stained with Bielschowsky silver nitrate solution in a $37^{\circ} \mathrm{C}$ incubator for $30 \mathrm{~min}$, Bielschowsky ammonia silver solution for $20 \mathrm{~s}$, gold chloride solution for $3 \mathrm{~min}$, respectively. The images were randomly captured at $20 \times$ magnification (XI 71 Olympus, Tokyo, Japan).

\section{Immunohistochemical Analysis (IHC)}

IHC was performed using an SP link detection kit (ZSGB-BIO Technology, Beijing, China). Tissue sections were dewaxed and dehydrated and washed with PBS. Subsequently, the samples were boiled in a citrate buffer ( $\mathrm{pH}$ 6.0) for antigen retrieval and blocked using $5 \%$ normal goat serum at $37^{\circ} \mathrm{C}$ for $1 \mathrm{~h}$. The sections were then incubated at $4^{\circ} \mathrm{C}$ overnight with primary antibodies (1:200). After washing with PBS, the sections were then incubated with the corresponding secondary antibody for $30 \mathrm{~min}$. Finally, diaminobenzidine was used as the chromogen to visualize the immunocomplexes, and then the sections were counterstained with hematoxylin. Images of the random brain and spinal cord sections were captured at $40 \times$ magnification and $20 \times$ magnification, respectively (XI 71 Olympus, Tokyo, Japan).

\section{Immunofluorescence Staining (IF)}

The lumbar enlargements of spinal cords and the right hemispheres of brains tissues were embedded in OCT and cut into $10-\mu \mathrm{m}$ sections for IFC staining. After washing with PBS, tissue sections were blocked with $5 \% \mathrm{BSA}$ for $30 \mathrm{~min}$ at $37^{\circ} \mathrm{C}$, and then incubated overnight at $4^{\circ} \mathrm{C}$ with primary antibodies. After washing, the sections were incubated with secondary antibodies $\mathrm{Cy} 3$ goat antirabbit $\operatorname{IgG}(\mathrm{H}+\mathrm{L})$ or Alexa Fluor 488 goat anti-mouse IgG $(\mathrm{H}+\mathrm{L})$ (1:200) for $1 \mathrm{~h}$ at $37^{\circ} \mathrm{C}$, and nuclei were stained with $4^{\prime}, 6$ diamidino-2-phenylindole (DAPI). Fluorescent images were captured at $40 \times$ magnification with a fluorescence microscope.

\section{Quantitation of Oxidative Stress}

Dihydroethidium (DHE; Molecular Probes, Eugene, OR, USA) staining was used to detect ROS levels in the lumbar enlargements of spinal cords and the right hemispheres of brains tissues. The sections were dewaxed and dehydrated with an ethanol gradient, after washing with PBS ( $\mathrm{pH} 7.4)$, tissue sections were blocked using $5 \% \mathrm{BSA}$ for $30 \mathrm{~min}$ at $37^{\circ} \mathrm{C}$, and stained with $5 \mu \mathrm{mol} / \mathrm{L} \mathrm{DHE}$ (in PBS) for $30 \mathrm{~min}$ at $37^{\circ} \mathrm{C}$. The ROS levels in EAE mice, in the absence or presence of bixin, were likewise evaluated by DHE staining. Finally, fluorescence images of brain or spinal cord sections were randomly captured at $20 \times$ magnification (XI 71 Olympus, Tokyo, Japan), and the fluorescent intensity was analyzed by Image J software.

\section{Cytokine Quantification via ELISA}

Peripheral blood was collected and centrifuged at 3,000 rpm for $20 \mathrm{~min}$, a portion of the serum was used for quantitating IL-1 $\beta$, IL-18, IL-17, and IFN- $\gamma$ levels by ELISA, while the remaining serum maintained $-80^{\circ} \mathrm{C}$ for further analysis. The corresponding ELISA kits were used to assay IL- $1 \beta$, IL-18, IL-17, and IFN- $\gamma$ levels (MIBIO Biotechnology, Shanghai, China).

\section{Measurement of Superoxide Dismutase (SOD), Malondialdehyde (MDA)}

The remaining serum of each mouse was used to detect the activities of SOD and the levels of MDA (The Institute of Biological Engineering of Nanjing Jiancheng, Nanjing, China). The protocol was according to the manufacturer's instructions.

\section{Flow Cytometry}

The CNS tissues and spleens were collected and mononuclear cells (MNCs) were isolated as previously reported $(23,24)$. CNS tissues were digested in dulbecco's modified eagle medium (DMEM) containing $2 \mathrm{mg} / \mathrm{ml}$ collagenase II (SigmaAldrich, St. Louis, MO, USA) and $1 \mathrm{mg} / \mathrm{ml}$ DNAseI (SigmaAldrich, St. Louis, MO, USA) at $37^{\circ} \mathrm{C}$ for $30 \mathrm{~min}$; MNCs were 
prepared by passing the tissue through a $100 \mu \mathrm{m}$ cell strainer and density gradient centrifugation using Percoll (30/70\%)(SigmaAldrich, St. Louis, MO, USA); MNCs were collected from the interface, washed, and resuspended in culture medium for further analysis. Splenocytes were isolated from the spleens of mice after being homogenized and passed through a $100 \mu \mathrm{m}$ cell strainer. To measure the percentages of Th1 and Th17 cells, MNCs were stimulated with $50 \mathrm{ng} / \mathrm{ml} \mathrm{PMA,} 500 \mathrm{ng} / \mathrm{ml}$ ionomycin containing GolgiPlug ${ }^{\mathrm{TM}}$ (BD Biosciences, San Jose, CA, USA) for $4 \mathrm{~h}$ at $37^{\circ} \mathrm{C}$, then permeabilized with Perm/Fix solution, next stained with IL-17 and IFN- $\gamma$ antibody at $4^{\circ} \mathrm{C}$ for $30 \mathrm{~min}$, respectively; finally, the cells were stained with CD4 antibody at $4^{\circ} \mathrm{C}$ for $30 \mathrm{~min}$. Cells were analyzed by BD Accuri C6 Flow cytometry (BD Biosciences, San Jose, CA, USA) and the data was analyzed by BD Accuri C6 software (BD Biosciences, San Jose, CA, USA).

\section{Quantitative Reverse-Transcription PCR (qRT-PCR)}

Total RNA was extracted from the spinal cords tissue using a total RNA extraction kit (Solarbio, Beijing, China) according to the manufacturer's instructions. Next, cDNA was synthesized using an iScript cDNA synthesis kit (Bio-Rad, Hercules, CA, USA). Nrf2, Cat, Nqo1, Txnip, Nlrp3, Asc, Casp1, Il18, Il1 $\beta$, Il6, $I l 8, I l 10, T n f \alpha, I l 17, I f n \gamma$, and Sod 2 mRNA levels were analyzed by qRT-PCR with SYBR Green Supermix (Bio-Rad, Hercules, CA, USA). The primers were synthesized by Shanghai Shenggong and are listed in Table $\mathbf{1}$ (Actb was used as an internal control for quantitation). The $2^{-\Delta \Delta C T}$ method was used to calculate relative mRNA levels.

\section{Western Blot Assay}

The spinal cords tissues were lysed in ice-cold RIPA lysis buffer (Beyotime Biotechnology, Shanghai, China). The protein concentration was determined using a BCA reagent kit (Beyotime Biotechnology, Shanghai, China). Total protein
(30 $\mu \mathrm{g}$ ) was separated by $10 \%$ sodium dodecyl sulfatepolyacrylamide gel electrophoresis, and transferred onto PVDF membranes (Millipore, Billerica, MA, USA). The membranes were blocked in tris-buffered saline with $5 \%$ non-fat milk and $0.5 \%$ bovine serum albumin for $1 \mathrm{~h}$ at room temperature and then incubated overnight at $4^{\circ} \mathrm{C}$ with primary antibodies $(1: 1,000)$. After washing, the membranes were incubated with secondary antibodies $(1: 5,000)$ for $1 \mathrm{~h}$ at $37^{\circ} \mathrm{C}$. Blots were visualized with the Chemiluminescent HRP substrate (Millipore) and quantified with the Quantity 5.2 software System (Bio-Rad).

\section{Statistical Analyses}

All data are expressed as mean \pm SD. Statistical analysis was performed using GraphPad Prism 7.0 software (GraphPad, San Diego, CA, USA) with one-way ANOVA, followed by post-hoc multiple comparisons with the Tukey's test. Statistical significance was considered as $\mathrm{P}<0.05$.

\section{RESULTS}

\section{Bixin Attenuates the Symptoms of EAE Mice}

To evaluate the effects of bixin on the symptoms of EAE mice (Figures 1A, B), daily weight changes and clinical behavioral scores were recorded. The results showed that bixin treatment significantly delayed the detrimental effects of EAE on body weight (Figure 1C). Furthermore, bixin treatment dosedependently reduced the clinical symptoms in the EAE mice (Figure 1D). At the same time, we found that EAE mice showed severe clinical signs with flaccid tail and complete paralysis of the hindlimbs; while, bixin-treated EAE mice only showed tail paralysis (Figure 1E). These results indicated that the appropriate dose of bixin to attenuate the symptoms of EAE was $100 \mathrm{mg} / \mathrm{kg}$, which was used for subsequent experiments.

TABLE 1 | Primer sequence information.

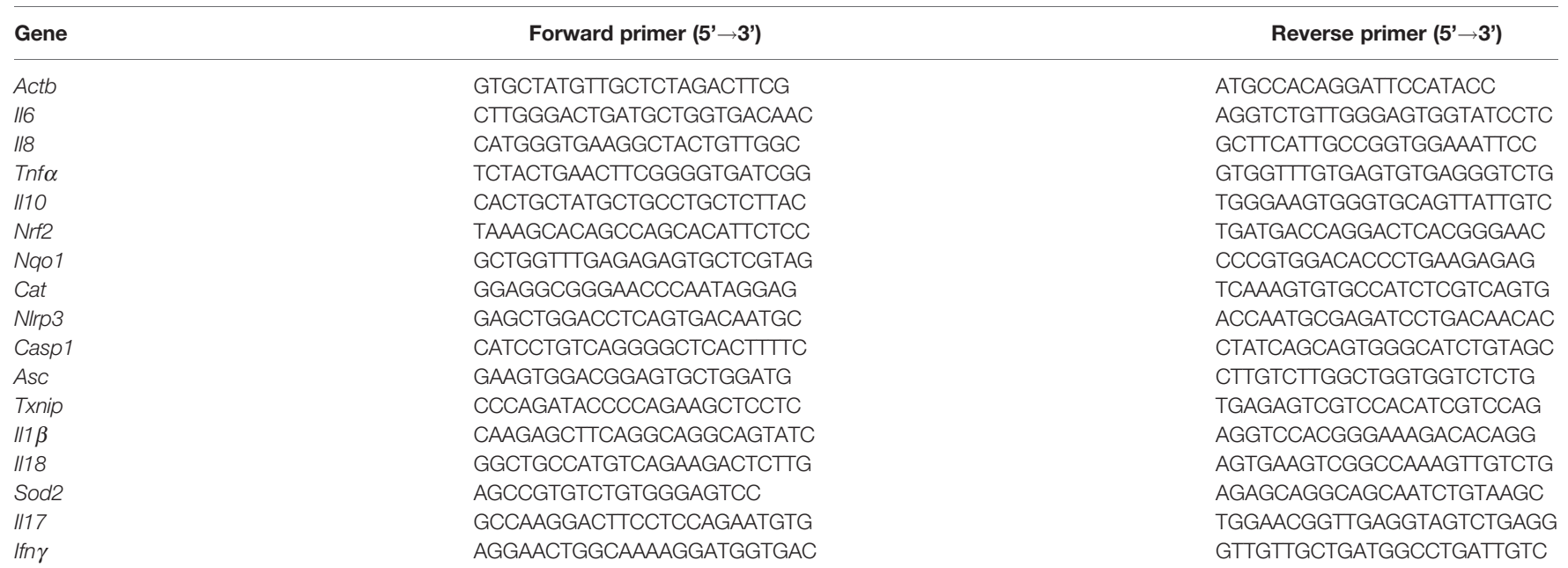


A

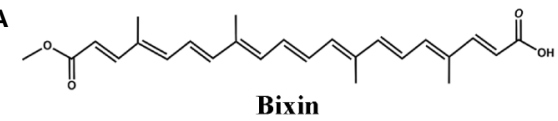

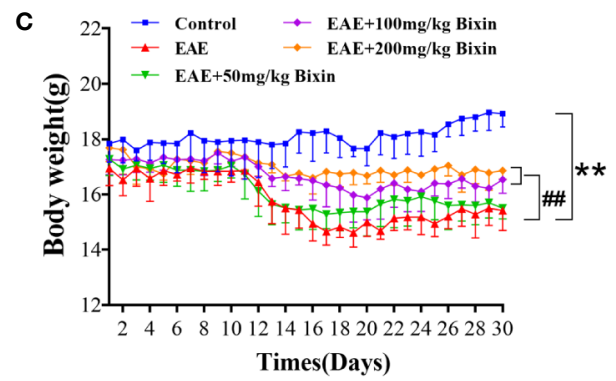

$\mathbf{E}$

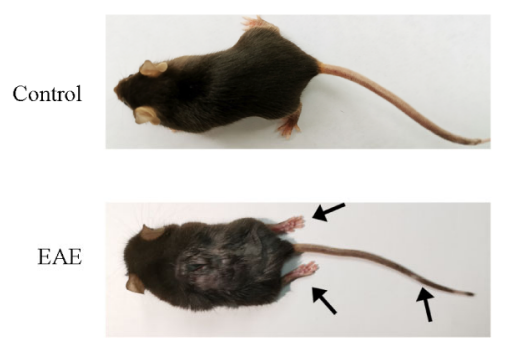

B
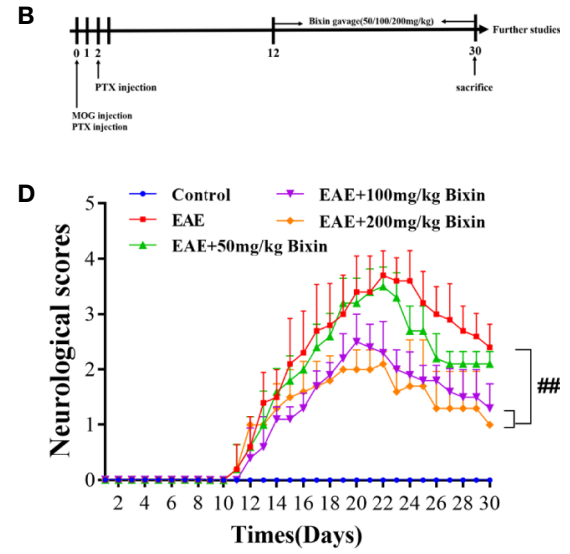

Bixin

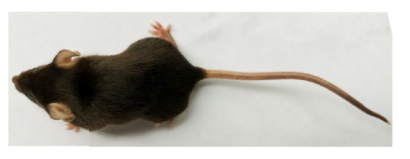

EAE+Bixin

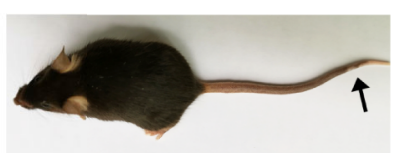

FIGURE 1 | Bixin attenuates the symptoms of EAE mice (A). Chemical structure of bixin (B). Study procedure (C). Body weight and (D) clinical behavior scores of the EAE mice (E). Representative images show behavioral symptoms of EAE mice in each group. Black arrows present limp tail or hind limb paralysis. Data shown in graphs represents the means $\pm S D(n=5)$. ${ }^{\star *} P<0.01$, vs. control group; ${ }^{\# \#} P<0.01$, vs. EAE group.

\section{Bixin Reduces the Inflammation and Demyelination in EAE Mice}

Previous studies have indicated that inflammation and demyelination are the primary features in EAE mice (5). Our results showed that EAE significantly increased the number of inflammatory cells and inflammation scores in the brain, however, bixin treatment noticeably reduced both of these effects (Figure 2A). Since the multiple inflammatory cells in the CNS which is important in MS study. Microglia are the main mediators of neuronal inflammation (25) and the lysosomal protein CD68 is highly expressed on the surface of activated microglia (5), we evaluated the co-localized expression of IBA1 and CD68 by IF. In line with the above results, although EAE significantly upregulated the levels of IBA1 and CD68, bixin treatment remarkably suppressed this phenotype (Figure 2B). And we detected the expression of $\mathrm{CD} 3+$ cells by IHC, the results showed that EAE significantly increased the expression of $\mathrm{CD} 3+$ cells, bixin significantly decreased the expression of $\mathrm{CD} 3+$ cells (Figure 2C).

Given that inflammatory cytokines play key roles in inflammation, we also evaluated the levels of several cytokines by qRT-PCR in the spinal cords and found that the mRNA levels of pro-inflammatory cytokinesr TNF- $\alpha$, IL-6, and IL-8, were markedly increased in the spinal cords of EAE mice, while that of the anti-inflammatory cytokine IL-10 was decreased. Meanwhile, bixin treatment reduced the mRNA levels of TNF- $\alpha$, IL- 6 , and IL-8, and increased that of IL-10 (Figures 2D-G).
Furthermore, LFB staining revealed that bixin treatment reduced the level of spinal cord demyelination in EAE mice (Figure 2H). Similarly, IF staining showed that bixin-treated EAE mice had significantly increased expression of MBP, a structural protein of myelin (Figure 2I). Meanwhile, we determined the degree of axon degeneration in the CNS by Bielschowsky staining. The results showed that bixin treatment significantly decreased the degree of axon degeneration (Figure 2J).

\section{Bixin Reduces the Percentages of Th1 and Th17 Cell Subsets}

Previous studies have demonstrated that T helper (Th) 1 and Th17 cells are responsible for the inflammatory demyelination in both MS and EAE. Th 1 cells primarily produce proinflammatory cytokines IFN- $\gamma$, while Th 17 cells secrete IL-17 $(24,26)$. Therefore, we evaluated the proportions of Th1 and Th17 cell subsets in the spleen and CNS by flow cytometry. Both Th1 and Th17 cell subsets in the spleen and CNS of EAE mice were significantly increased compared to the control group, while bixin treatment remarkably reduced the proportion of both cell subsets (Figures 3A, B). Meanwhile, we quantified the IFN- $\gamma$ and IL-17 levels by qRT-PCR and ELISA, and found that EAE significantly upregulated the levels of IFN- $\gamma$ and IL-17, while bixin treatment remarkably reduced the expression of both (Figures 3C-F). 
A

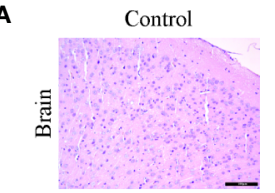

B
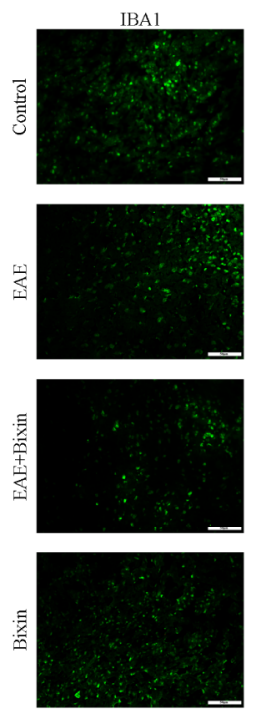

C
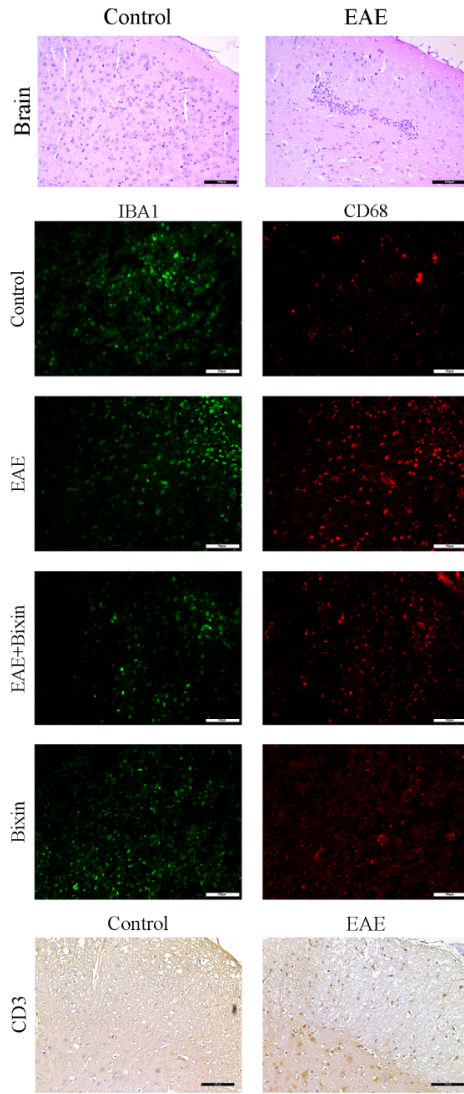

$\mathrm{CD} 68$
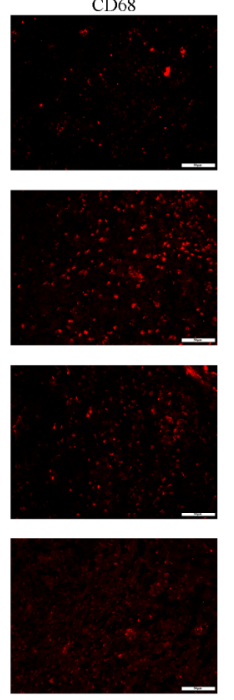

EAE
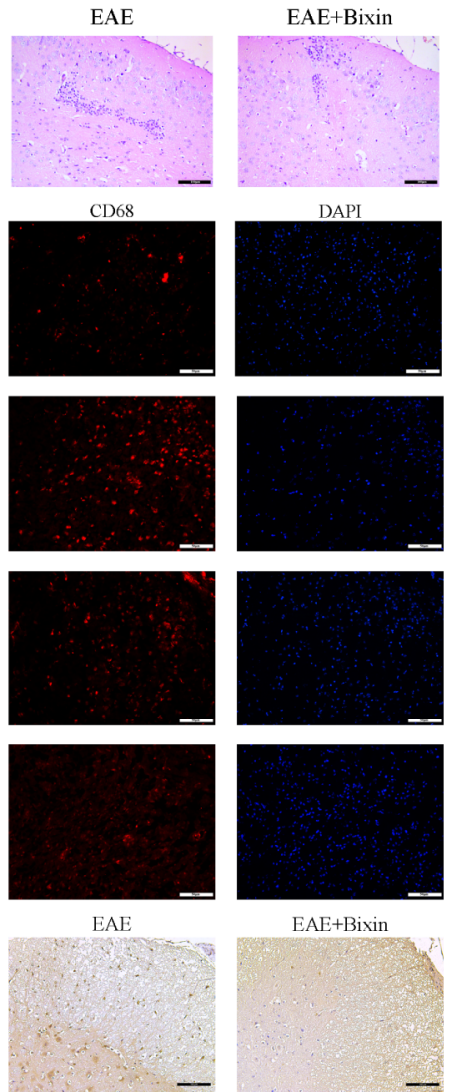

$\mathrm{EAE}+$ Bixin
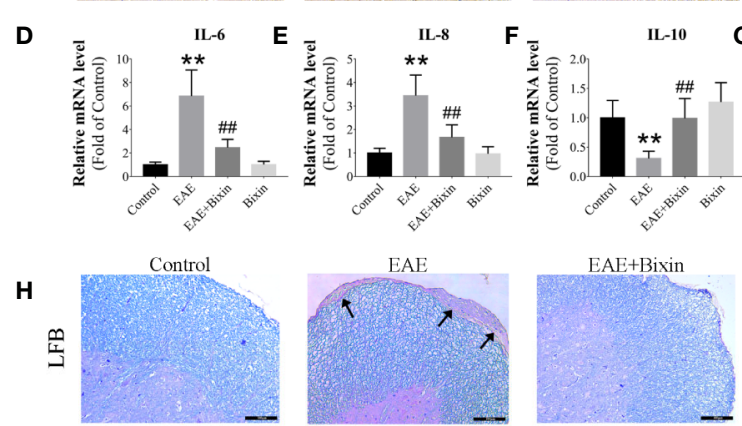

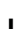

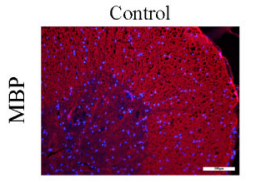

$\mathbf{J} \cdot$

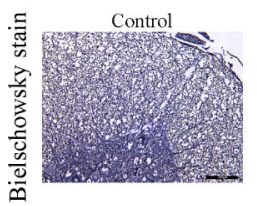

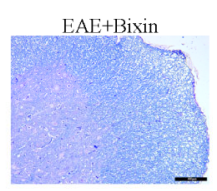
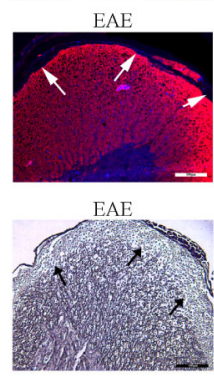

$\mathrm{EAE}+\mathrm{Bixin}$

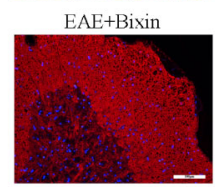

$\mathrm{EAE}+\mathrm{Bixin}$

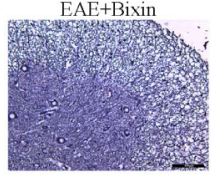

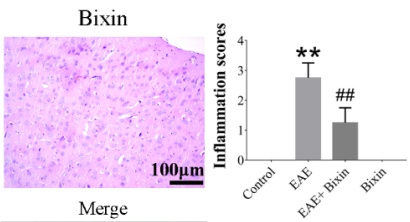
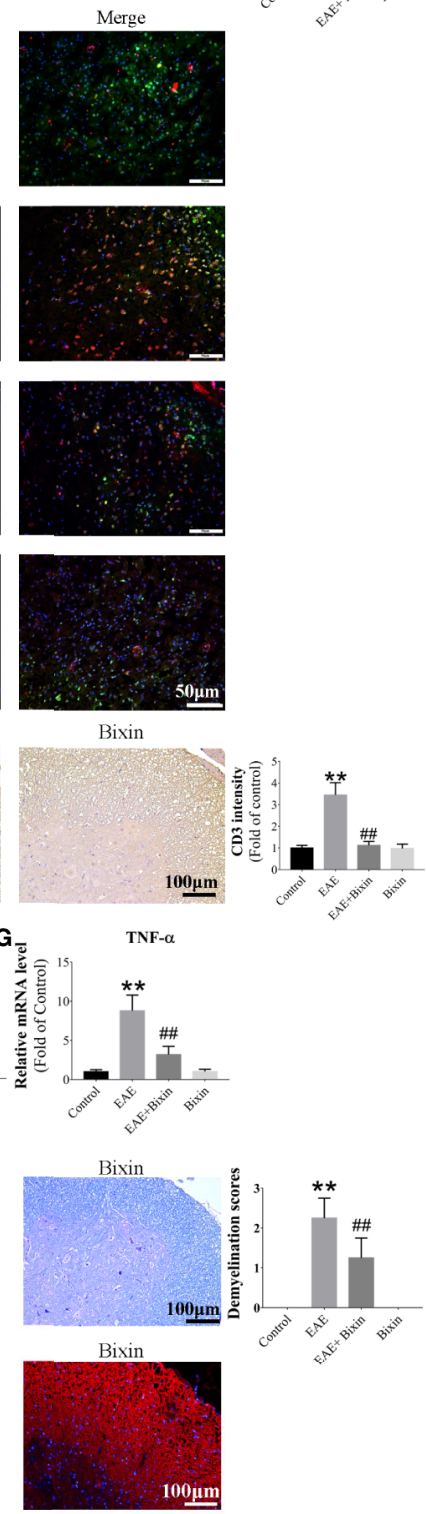

Bixin

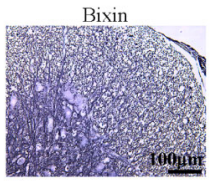

FIGURE 2 | Bixin reduces the inflammation and demyelination in EAE mice (A). H\&E staining showing inflammatory cell infiltration in the brain, as well as the inflammation scores. Scale bars: $100 \mu \mathrm{m}$ (B). Representative IF images of IBA1 and CD68 in brain sections showing the activated microglia. Scale bars: $50 \mu \mathrm{m}$ (C). Representative IHC images of CD3+ cells in the spinal cord. Scale bars: $100 \mu \mathrm{m}$ (D-G). mRNA expression of the inflammatory cytokines IL-6, IL-8, IL-10, and TNF- $\alpha$ were quantitated by qRT-PCR (H). LFB staining showing demyelination in the spinal cord, as well as the demyelination scores. Scale bars: $100 \mu \mathrm{m}(\mathbf{I})$. IF staining of MBP in the spinal cords. Scale bars: $100 \mu \mathrm{m}$ (J). Bielschowsky staining showing the degree of axon degeneration. Scale bars: $100 \mu \mathrm{m}$. Data shown in graphs represents the means $\pm S D(n=5)$. ${ }^{*} P<0.01$, vs. control group; ${ }^{\# \#} P<0.01$, vs. EAE group. 


\section{A Spleen}
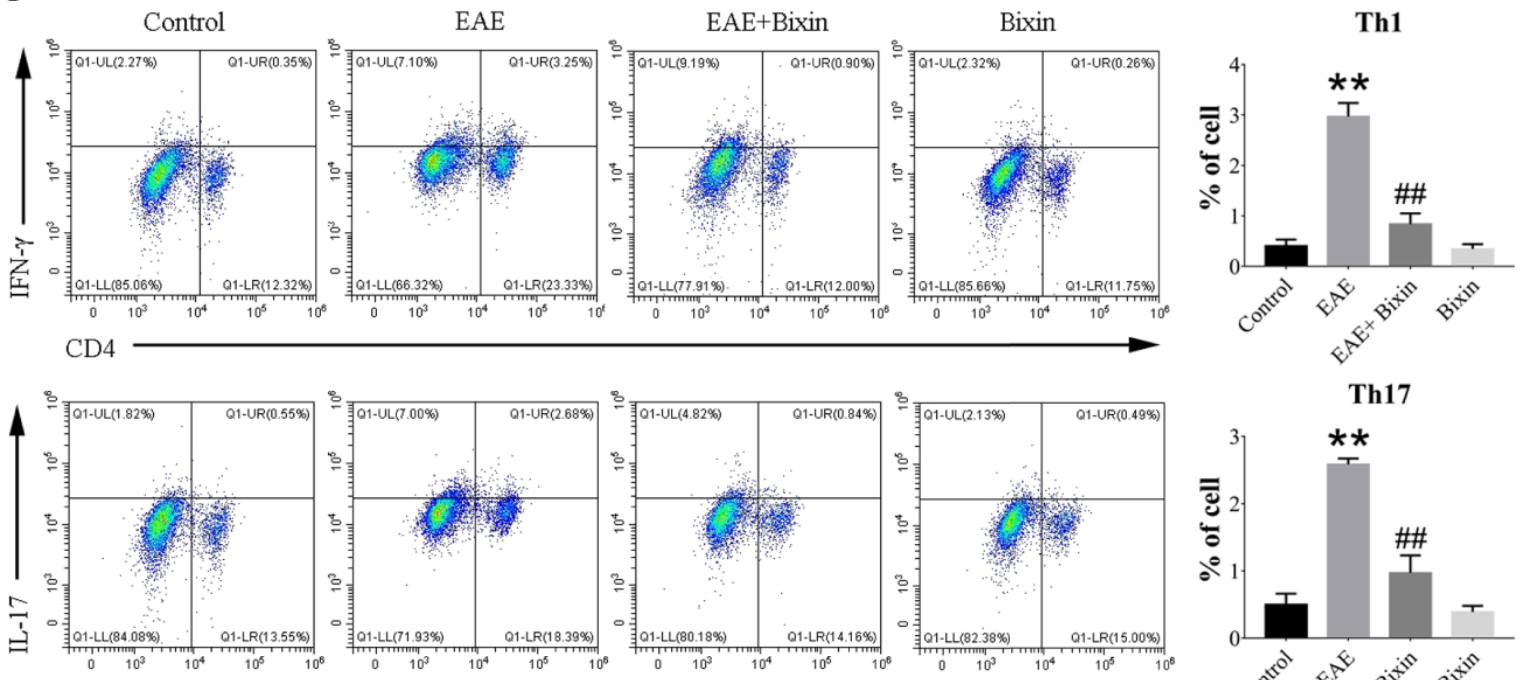

CD4

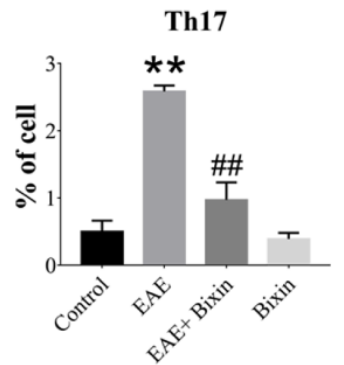

B CNS
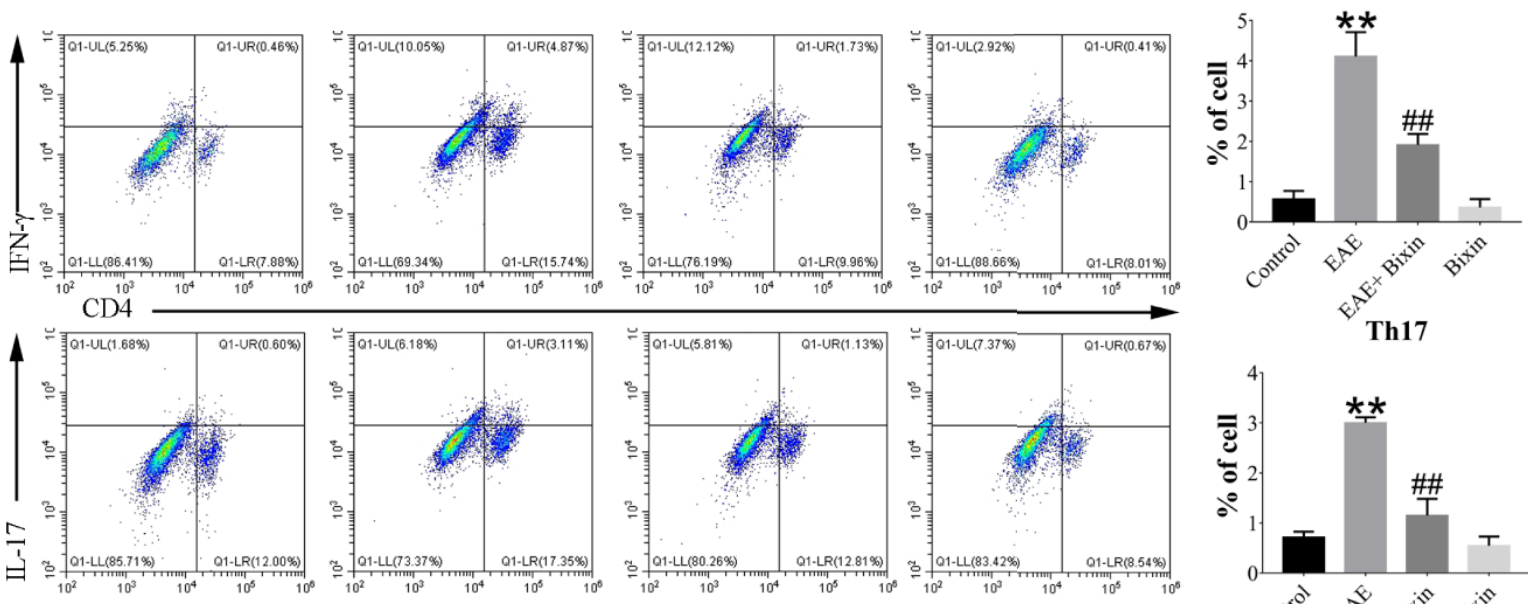

Th17
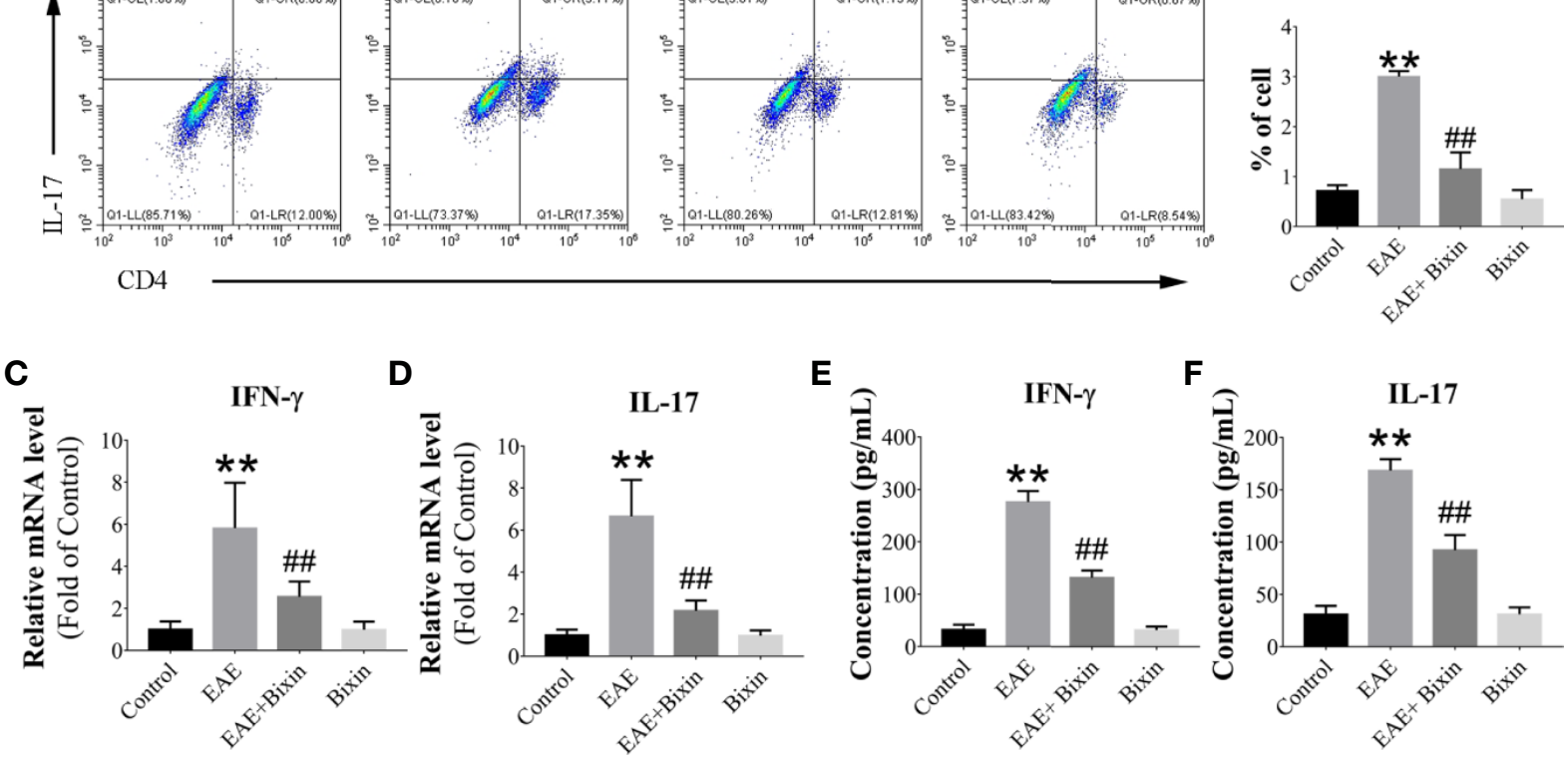

FIGURE 3 | Bixin reduces the proportion of Th1 and Th17 cell subsets. Subsets of Th1 and Th17 cells in CD4+ gate were analyzed by intracellular staining for IFN- $\gamma$ and IL-17, respectively, in MNCs from the spleen $\mathbf{( A )}$ and CNS (B). (C, D). mRNA expression of the inflammatory cytokines IFN- $\gamma$ and IL-17 were quantitated by qRT-PCR (E-F). IFN- $\gamma$ and IL-17 levels in serum were measured by ELISA. Data shown in graphs represents the means \pm SD $(n=5)$. ${ }^{\star \star} P<0.01, \boldsymbol{v s}$. control group; ${ }^{\# \#} \mathrm{P}<0.01$, vs. EAE group. 


\section{Bixin Suppresses Activation of the TXNIP/ NLRP3 Inflammasome in EAE Mice}

To clarify whether bixin suppresses the inflammatory response in EAE mice by inhibiting the activation of the TXNIP/NLRP3 inflammasome, we evaluated the individual protein component levels for this complex in the spinal cords by western blotting. The results showed that TXNIP, NLRP3, ASC, caspase-1, IL-1 $\beta$, and IL-18 levels were increased in EAE mice, and bixin treatment suppressed this effect (Figure 4A). The same results were observed for the mRNA levels of these components by qRTPCR (Figures 4B-G). Furthermore, IL-1 $\beta$ and IL-18 levels in serum were increased in EAE mice, and bixin treatment reduced their upregulation (Figures $\mathbf{4 H}, \mathbf{I}$ ). These findings were corroborated by IHC; although NLRP3 were highly expressed in the CNS of EAE mice, bixin treatment remarkably inhibited their expression (Figures 4J, K).

\section{Bixin Inhibits Oxidative Stress in EAE Mice}

To elucidate whether the anti-inflammatory effect of bixin occurred through an anti-oxidative effect, we evaluated the ROS levels in the brain and spinal cord by DHE staining. The results showed that ROS levels were significantly increased in EAE mice, and bixin treatment alleviated this oxidative stress (Figure 5A). Additionally, western blotting results showed that
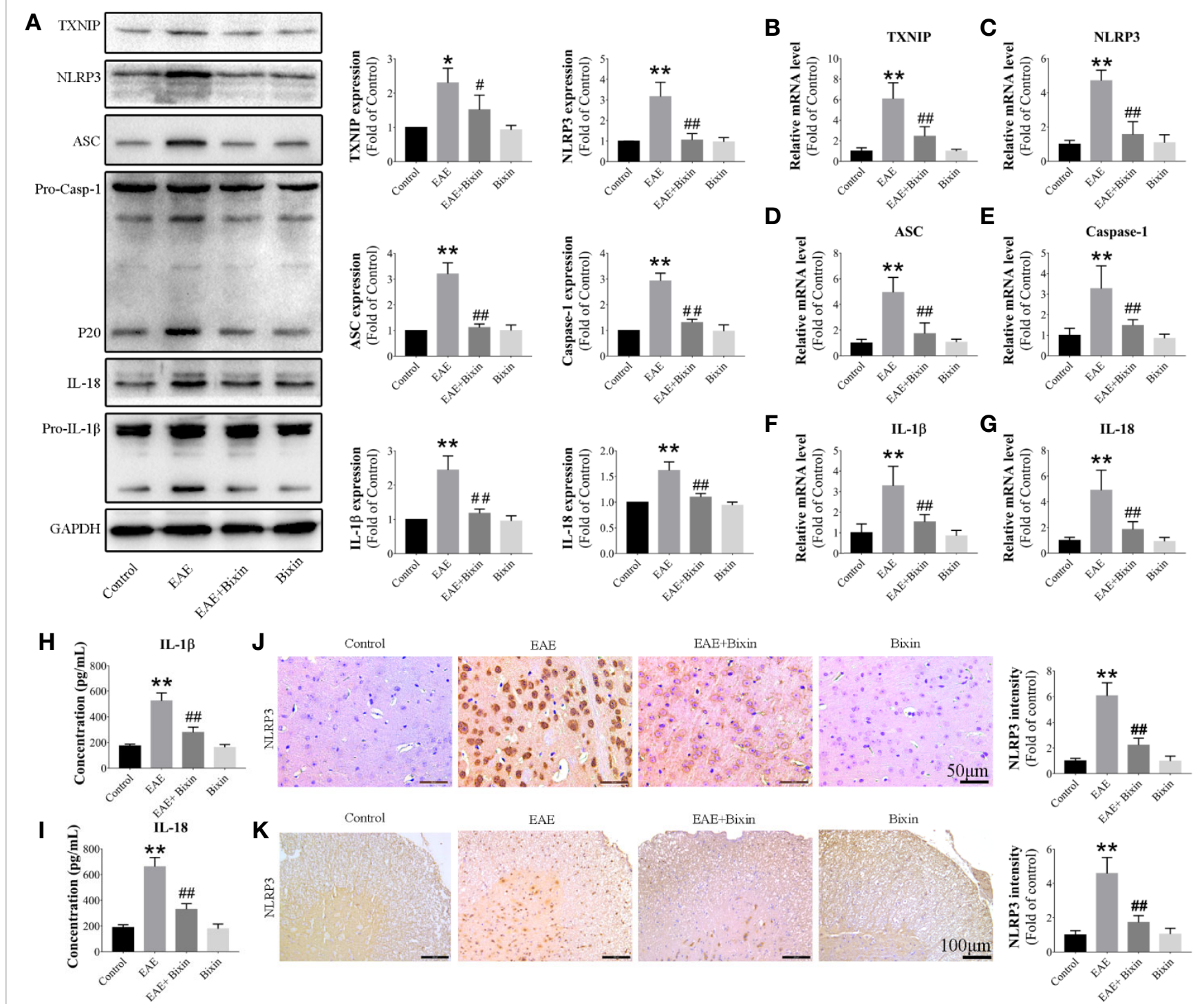

FIGURE 4 | Bixin suppresses the activation of the TXNIP/NLRP3 inflammasome in EAE mice (A). The protein levels of TXNIP/NLRP3 inflammasome components were assessed by western blotting (B-G). mRNA expression of TXNIP/NLRP3 inflammasome components were quantitated by qRT-PCR (H, I). IL-1 3 and IL-18 levels in serum were measured by ELISA. Representative IHC images of NLRP3 in brain sections (J) (Scale bars: $50 \mu \mathrm{m})$ and spinal cord sections (K) (Scale bars: $100 \mu \mathrm{m})$, and the quantitative results are shown. Data shown in graphs represents the means $\pm \mathrm{SD}(\mathrm{n}=5)$. ${ }^{*}<0.05$, ${ }^{\star *} \mathrm{P}<0.01$, vs. control group; ${ }^{\#} \mathrm{P}<0.05$, $\# P<0.01$, vs. EAE group. 
A

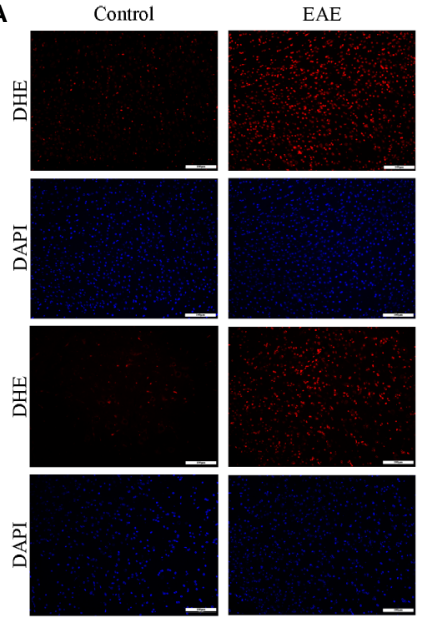

B

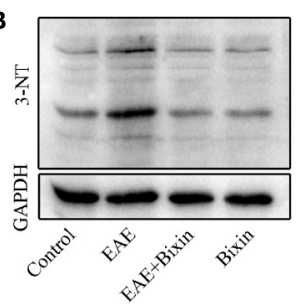

EAE+Bixin
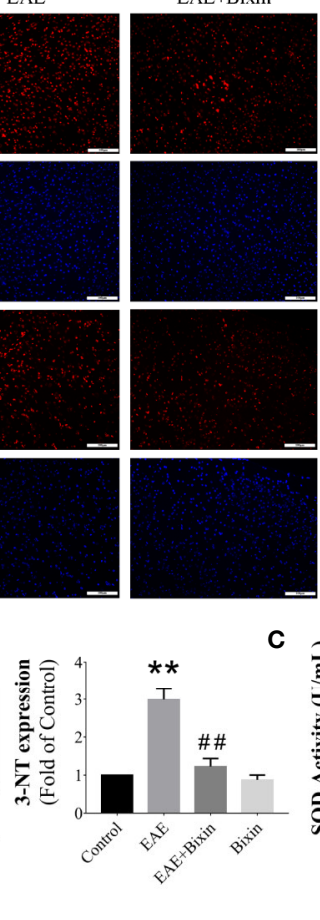

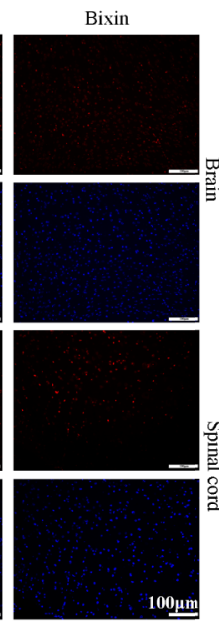

C

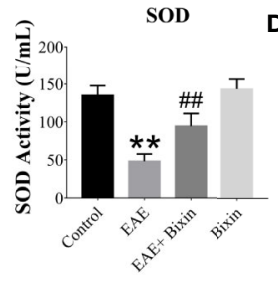

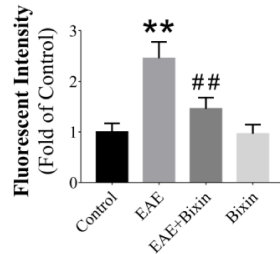
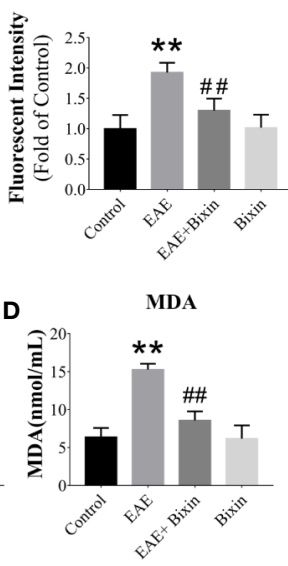

FIGURE 5 | Bixin alleviates oxidative stress in EAE mice (A). The level of reactive oxygen species (ROS) in the brain and spinal cord tissues was assessed by DHE staining, and the quantitative results are shown. Scale bars: $100 \mu \mathrm{m}$ (B). The protein level of 3-NT in the CNS tissues was assessed by western blotting, and the quantitative results are shown (C). The activities of SOD and (D) the levels of MDA in the serum. Data shown in graphs represents the means $\pm \operatorname{SD}(n=5)$. ${ }^{\star \star} P<0.01$, vs. control group; ${ }^{\# \#} P<0.01$, vs. EAE group.

the level of the oxidative damage marker 3-NT in EAE mice was reduced by bixin treatment (Figure 5B). Furthermore, bixin treatment reduced the level of $\mathrm{MDA}$, while increasing the expression of SOD in EAE mice (Figures 5C, D).

\section{Bixin Activates the NRF2 Signaling in EAE Mice}

Since NRF2 plays a critical role in oxidative stress response (27), we next assessed the effect of bixin on NRF2. To this end, we evaluated the levels of NRF2 signaling components by western blotting and found that although the levels of NRF2, and its downstream proteins, catalase, NQO-1, and SOD2 were reduced in EAE mice, bixin treatment significantly upregulated their expression (Figure 6A). Similarly, the mRNA levels had the same trends in expression (Figures 6B-E). Furthermore, IHC indicated that NRF2 expression was significantly reduced in EAE mice, and bixin treatment reversed this phenotype (Figures 6F, G).

To confirm the pivotal role of NRF2 in the bioactivity of bixin, the NRF2 inhibitor ML385 was intraperitoneally administered $1 \mathrm{~h}$ before intragastric administration of bixin, which significantly decreased the expression of NRF2 and its downstream genes, and increased the levels of ROS (Figures 7A, B, E, F) and inflammation (Figures 7C, D, G, H). However, bixin could not reverse this phenotype in ML385-treated EAE mice (Figure 7).

\section{DISCUSSION}

MS is a characteristic autoimmune inflammatory demyelinating disease of the CNS that significantly impacts the quality of life of those affected (28). While numerous drugs are available for MS treatment, their efficacy remains unsatisfactory. Hence, numerous researchers have sought to better understand the pathophysiology of this disease to inform the design of improved therapeutics. To this end, Dang C et al. (29) reported that upregulating PGC-1 $\alpha$ significantly improves the survival ability of neurons in EAE via inhibiting oxidative stress. Additionally, ghrelin decreases neuroinflammation and demyelination by inhibiting the NLRP3 inflammasome activity (5). In this study, we similarly demonstrated that NLRP3 inflammasome activity and oxidative stress were the key factors in EAE development.

Bixin is a natural carotenoid with multiple bioactivities. Previous studies have shown that bixin has anti-inflammation, anti-tumor, and anti-oxidative effects (30-34). Xu Z et al. (19) have found that bixin attenuates cardiac injury by inhibiting inflammation and oxidative stress in a high-fat-diet mouse model. In this study, we found that bixin, a natural carotenoid, suppressed inflammatory cell infiltration and the levels of TNF$\alpha$, IL-6, IL- 8, IL-17, and IFN- $\gamma$, while upregulating the expression of IL-10. Microglia are resident immune effector cells in the brain that are easily activated resulting in chronic neuroinflammatory 
A

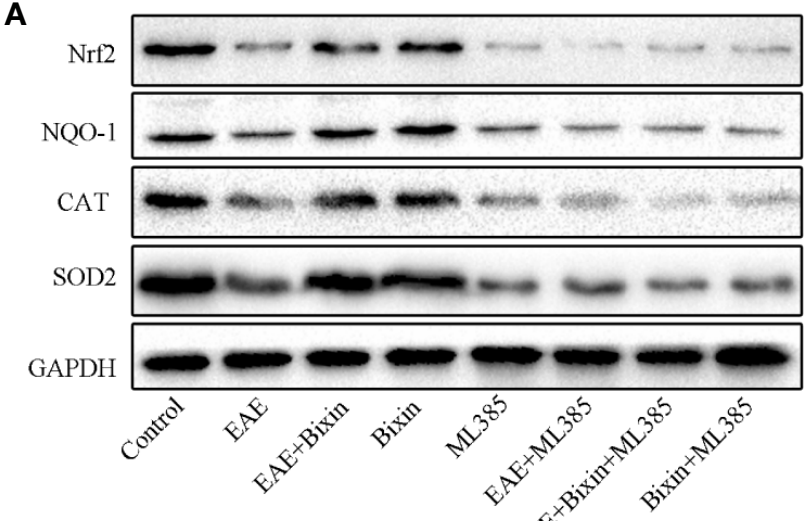

B

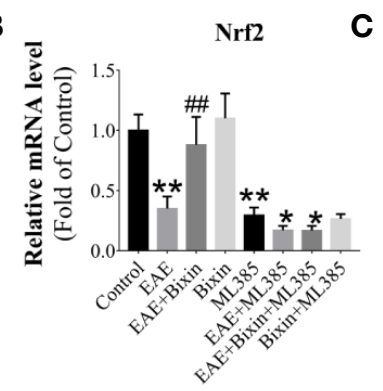

$\mathbf{F}$

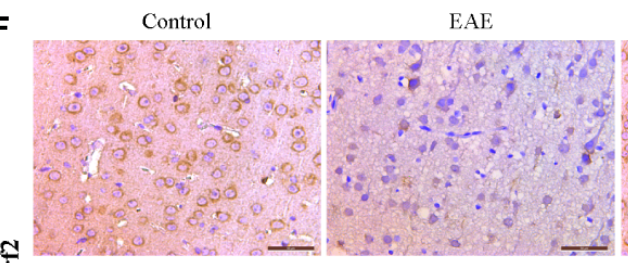

$\frac{1}{\mathrm{Z}}$

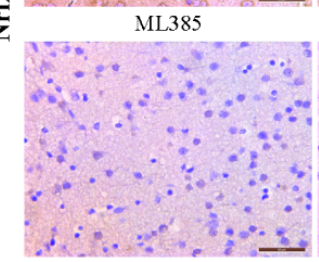

G
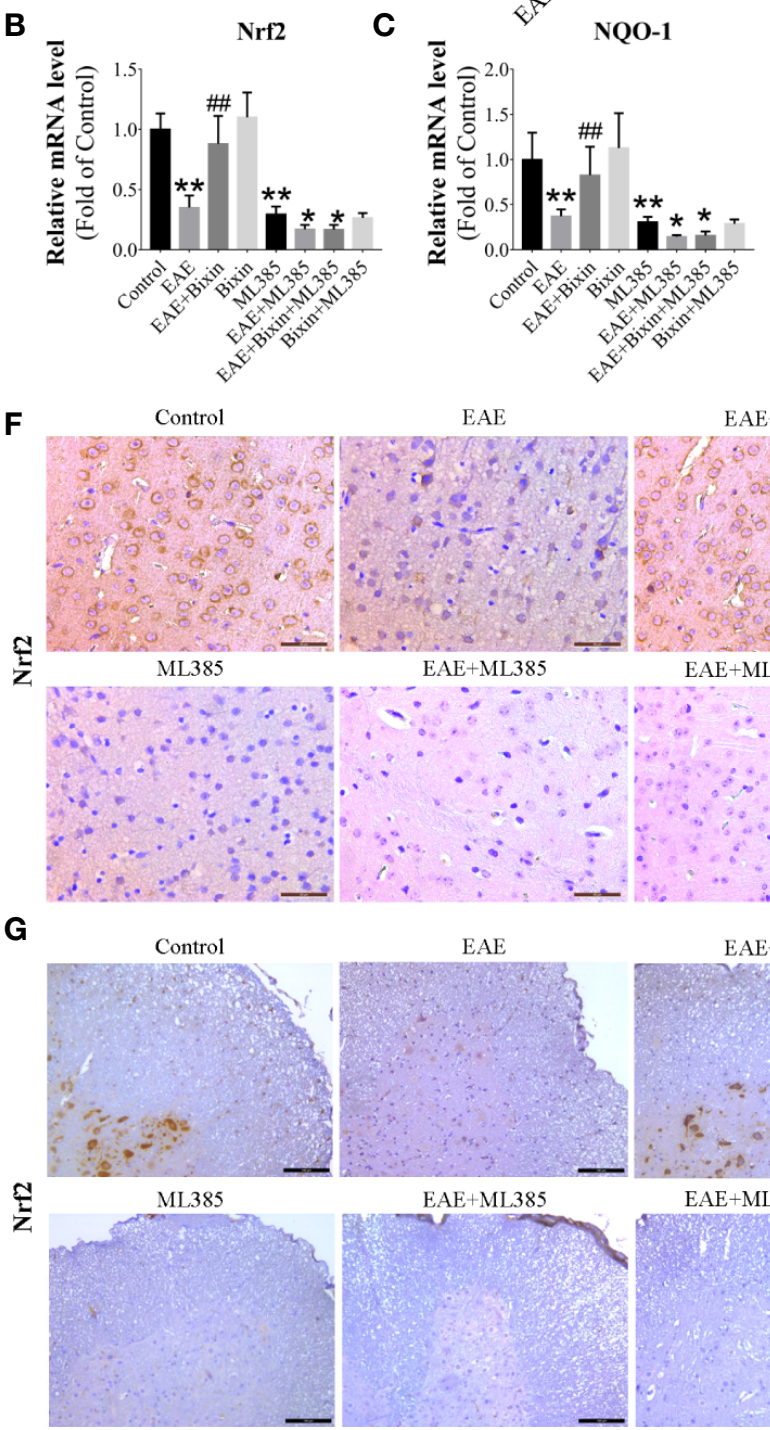

$\mathrm{EAE}+\mathrm{Bixin}$

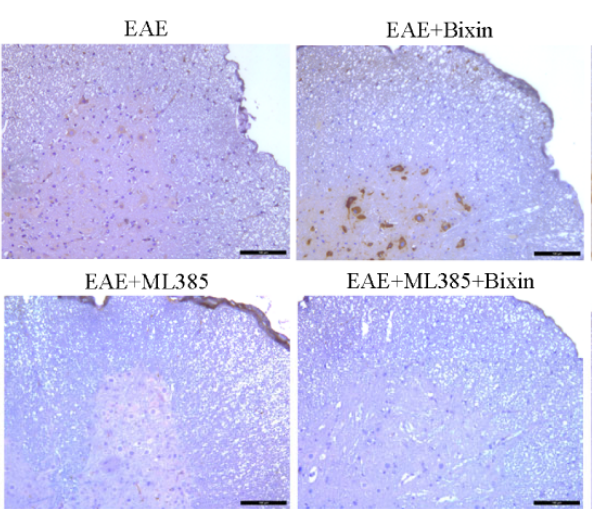

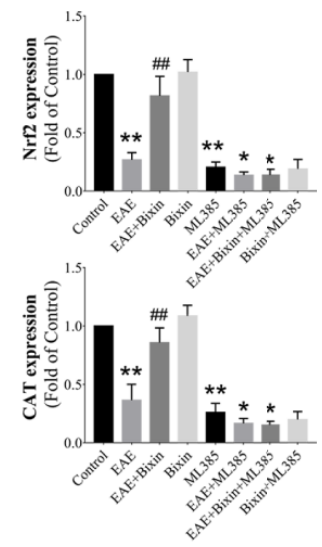
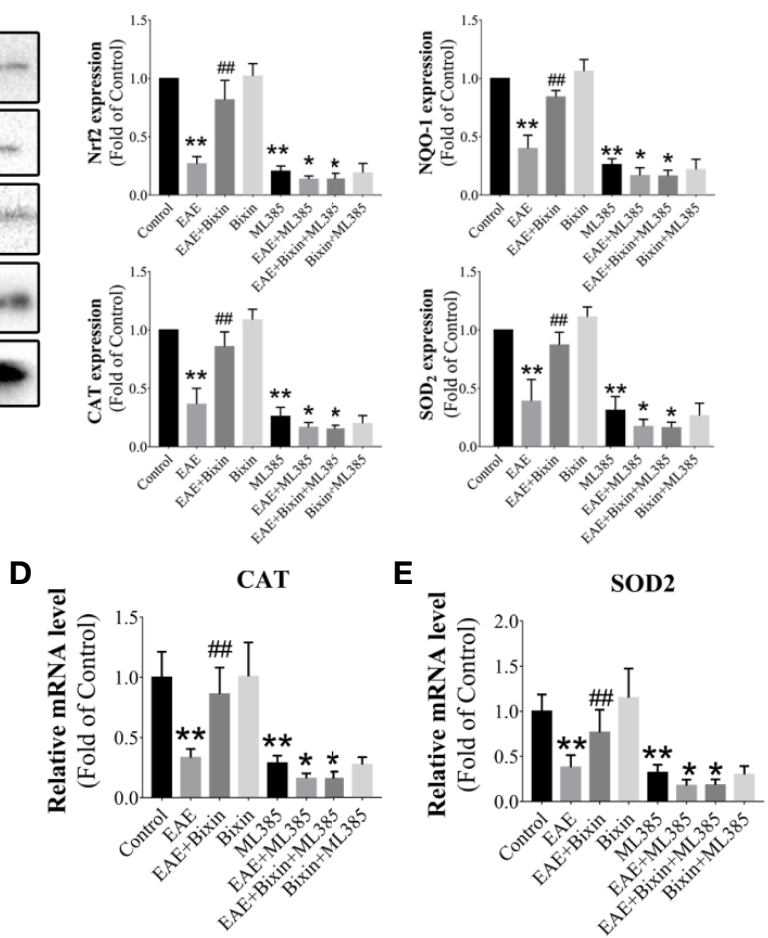

Bixin
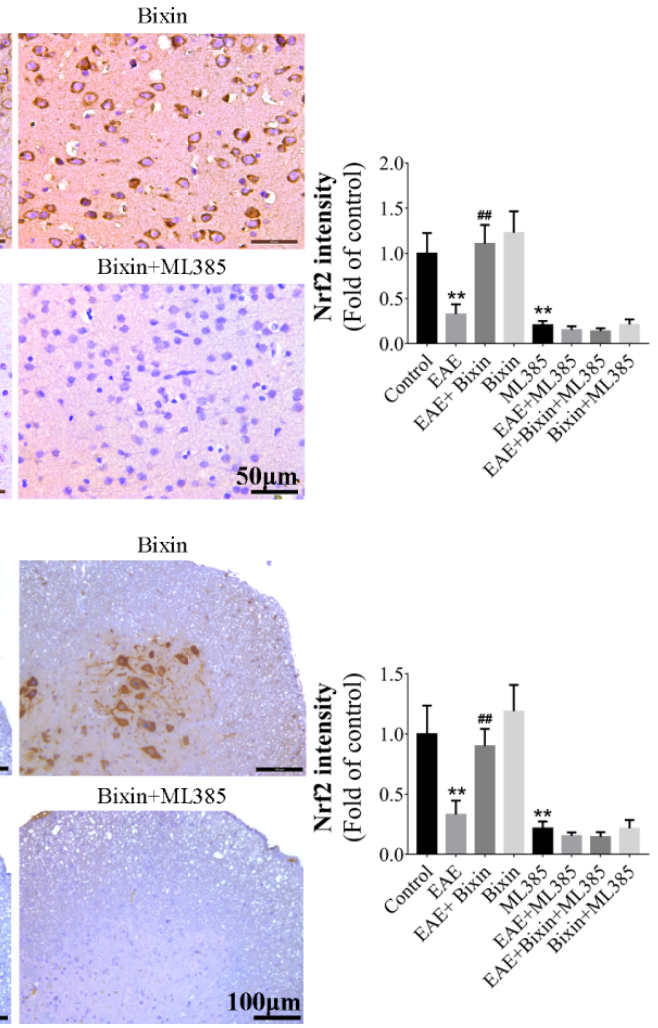

FIGURE 6 | Bixin activates the NRF2 signaling in EAE mice (A). The protein levels of NRF2 and downstream genes Catalase, NQO-1, and SOD2 in the brain tissues were assessed by western blotting, and the quantitative results are shown (B-E). mRNA expression of NRF2, Catalase, NQO-1, and SOD2 in the CNS tissues were quantitated by qRT-PCR. IHC of NRF2 in brain sections (F) (Scale bars: $50 \mu \mathrm{m}$ ) and spinal cord sections (G) (Scale bars: $100 \mu \mathrm{m}$ ). Data shown in graphs represents the means $\pm \mathrm{SD}(\mathrm{n}=5)$. ${ }^{\star} \mathrm{P}<0.05$, ${ }^{\star} \mathrm{P}<0.01$, vs. control group or ML385 group; ${ }^{\# \# ~} \mathrm{P}<0.01$, vs. EAE group or EAE $+\mathrm{ML385}$ group. 
A Brain
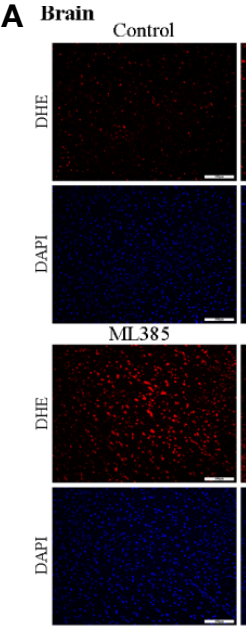

B Spinal cord
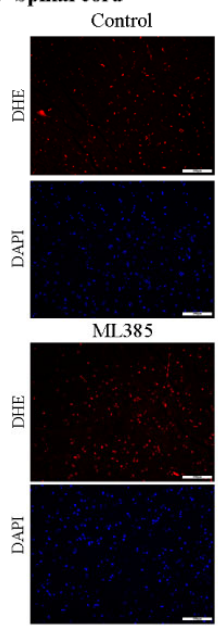

EAE
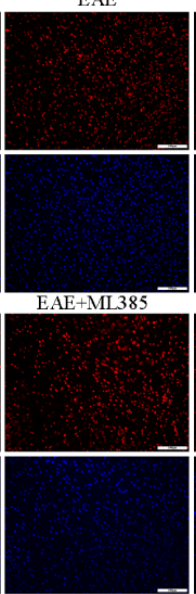

EAE
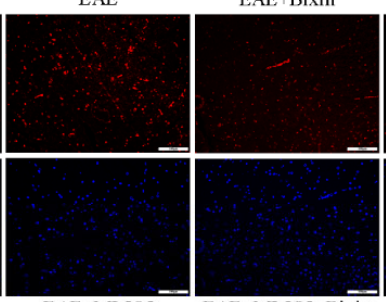

EAE+ML385

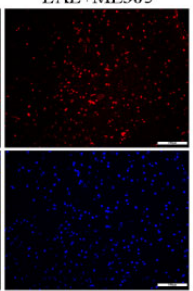

EAE + Bixin

Bixin
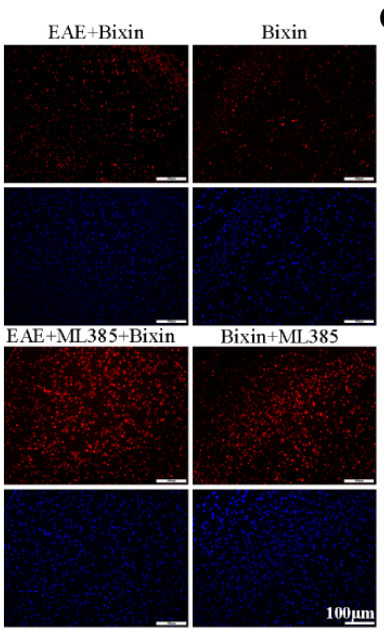

$100 \underline{\underline{m}}$
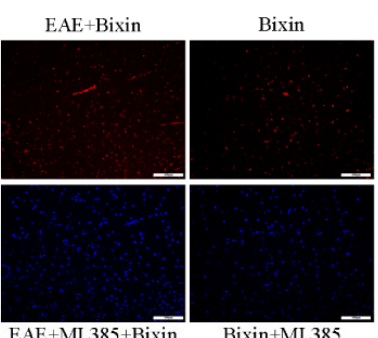

EAE+ML 385+Bixin
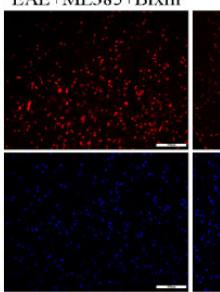

C Brain
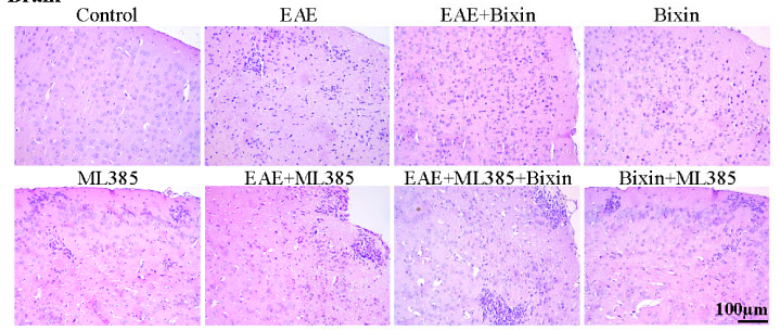

\section{Spinal cord}

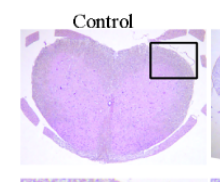

EAE

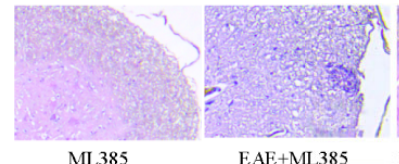

ML385

$\mathrm{EAE}+\mathrm{ML} 385$
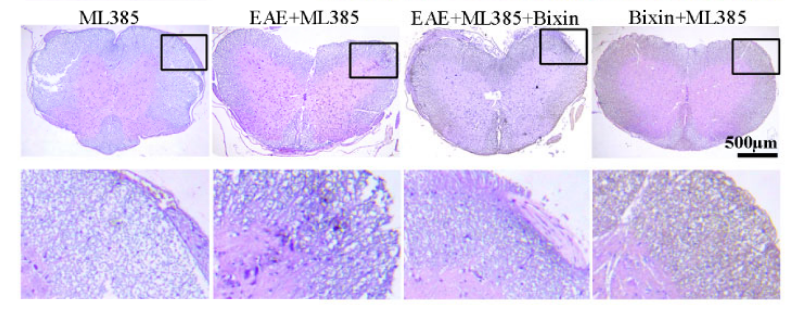

E
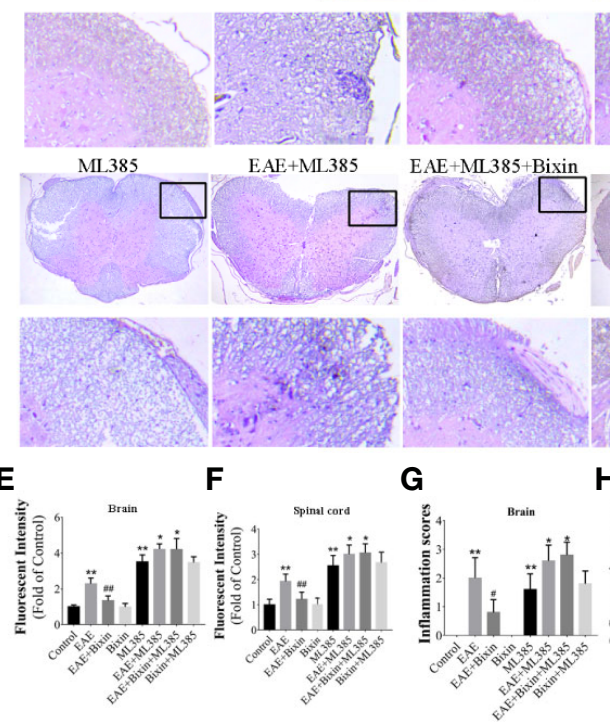

H
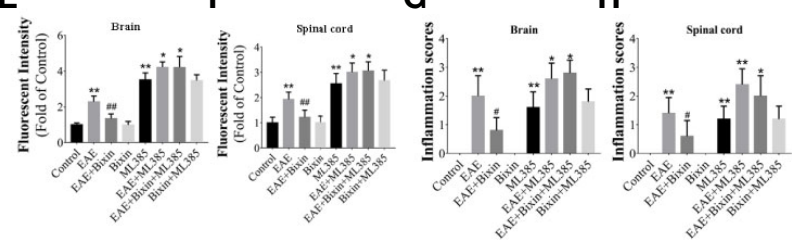

FIGURE 7 | Bixin suppressed the production of ROS and inflammation in EAE mice by activating NRF2 signaling. The level of reactive oxygen species (ROS) in the brain tissues (A) and spinal cord tissues (B) was assessed by DHE staining, as well as the quantitative results (E, F). Scale bars: $100 \mu \mathrm{m}$. H\&E staining showing inflammatory cell infiltration in the brain (C) (scale bars: $100 \mu \mathrm{m}$ ) and the spinal cord (D) (scale bars: $500 \mu \mathrm{m}$ ), as well as the inflammation scores (G, H). Data shown in graphs represents the means $\pm S D(n=5)$. ${ }^{*} P<0.05$, ${ }^{\star *} P<0.01$, vs. control group or ML385 group; ${ }^{\#} P<0.05$, ${ }^{\# \#} P<0.01$, vs. EAE group or $E A E+M L 385$ group.

reaction in the brain. What's more, these cells are closely associated with the development of neurodegenerative diseases, such as Alzheimer's disease $(31,35)$. In our study, we found that EAE upregulated the expression of IBA1 and CD68, markers of activated microglia; however, bixin was found to reduce microglia activation in the brain. And bixin significantly decreased the expression of CD3+ cells. These results indicated that bixin improved the symptoms in EAE mice and reduced demyelination by inhibiting inflammatory cell infiltration and the production of inflammatory cytokines.

Autoreactive pathogenic $\mathrm{T}$ lymphocytes have also been described as being closely related to the development and progression of MS and EAE (26). Specifically, activated CD4+ $\mathrm{T}$ cell subsets produce a large number of inflammatory mediators, such as ROS, which induces subsequent oxidative stress and inflammatory demyelination in EAE. Recent studies have found that CD4+ T subsets play key roles in MS and EAE pathogenesis, including Th1 cells, which primarily secrete proinflammatory cytokines IFN- $\gamma$, and Th17 cells, which produce IL-17 $(23,24,26,36)$. In our study, we found that EAE increased the proportion of Th1 and Th17 cell subsets in the spleen and CNS, resulting in corresponding increased levels of IL-17 and IFN- $\gamma$, while bixin treatment markedly inhibited these effects. These results indicated that bixin reduced neuroinflammation in EAE via an immune regulatory mechanism.

The TXNIP/NLRP3 inflammasome plays a key role in the pathogenesis of various diseases $(32,34,37,38)$. Chen W. et al. (9) indicated that minocycline improves diabetic retinopathy by inhibiting the TXNIP/NLRP3 inflammasome pathway, and vitamin D3 attenuates diabetic retinopathy by inhibiting high-glucose-induced ROS/TXNIP/NLRP3 inflammasome pathway (39). Emerging evidence suggests that the NLRP3 
inflammasome, composed of NLRP3, ASC, and caspase-1, plays a critical role in the pathogenesis of MS and EAE (40, 41). And recent researches have reported that NLRP3 inflammasome inhibitor JC-171 and OLT1177 were a potential therapeutic agent for MS $(34,42)$. Liu F et al. (5) found that Ghrelin attenuated MS by suppressing the activation of NLRP3 inflammasome. In the present study, we also found that the TXNIP/NLRP3 inflammasome components were upregulated in EAE mice, and bixin significantly downregulated the expression of TXNIP and NLRP3 at both the mRNA and protein levels. These results indicated that bixin reduced neuroinflammation in EAE by suppressing the activation of the TXNIP/NLRP3 inflammasome.

Recent studies have reported that oxidative stress is one of the main causes of CNS dysfunction in MS (27). ROS are also the main mediators of oxidative stress and initiators of the TXNIP/ NLRP3 inflammasome (43-45). In our study, we found that bixin attenuated ROS accumulation, downregulated the expression of oxidative damage marker 3-NT and the levels of MDA in EAE mice, and increased the expression of SOD. These results indicated that bixin suppressed the activation of the TXNIP/NLRP3 inflammasome through the attenuation of oxidative stress.

NRF2, an important redox sensor, binds to antioxidant response elements and counteracts the production of ROS by activating the expression of many antioxidant genes $(46,47)$. Meanwhile, bixin has been reported to alleviate photodamage and hair graying by activating NRF2 signaling (48); and to promote tissue repair and improve pulmonary injury induced by particle exposure in a NRF2-dependent manner $(20,49)$. In our study, the results showed that bixin markedly upregulated NRF2 and its downstream antioxidant target genes in EAE mice. These results indicated that bixin suppressed the production of ROS by activating NRF2 signaling. Furthermore, we found that the protective role of bixin was dependent on NRF2 activation, as the NRF2 inhibitor ML385 caused near complete abrogation of the effects elicited by bixin on ROS attenuation. Therefore, these findings indicated that bixin prevented neuroinflammation and demyelination in EAE mice primarily by scavenging ROS through activation of the NRF2 signaling pathway.

\section{CONCLUSIONS}

Bixin inhibits the TXNIP/NLRP3 inflammasome and activates the NRF2 signaling pathway in EAE mice. While bixin may be a possible therapeutic strategy for MS, further mechanistic studies in vitro are necessary.

\section{REFERENCES}

1. Williams SK, Fairless R, Maier O, Liermann PC, Pichi K, Fischer R, et al. AntiTNFR1 targeting in humanized mice ameliorates disease in a model of multiple sclerosis. Sci Rep (2018) 8:13628. doi: 10.1038/s41598-018-31957-7

2. De Paula ML, Rodrigues DH, Teixeira HC, Barsante MM, Souza MA, Ferreira AP. Genistein down-modulates pro-inflammatory cytokines and reverses

\section{DATA AVAILABILITY STATEMENT}

The datasets presented in this study can be found in online repositories. The names of the repository/repositories and accession number(s) can be found in the article/ Supplementary Material.

\section{ETHICS STATEMENT}

The animal study was reviewed and approved by the Medical Ethics Committee of Chengdu Medical College.

\section{AUTHOR CONTRIBUTIONS}

YY and D-MW researched data. YY and D-MW wrote, reviewed, and edited the manuscript. JL, S-HD, TL, TZ, MH, and Y-YZ provided research material and techniques. YX directed the project, and wrote, reviewed, and edited the manuscript. All authors contributed to the article and approved the submitted version.

\section{FUNDING}

This study was funded by the National Natural Science Foundation of China (81972977 and 81802955), Foundation of Sichuan Science and Technology Agency (2018JY0648 and 2019YJ0589), Foundation of The First Affiliated Hospital of Chengdu Medical College (CYFY2017ZD03 and CYFY2018ZD02), Foundation of Collaborative Innovation Center of Sichuan for Elderly Care and Health, Chengdu Medical College (19Z01), and the Foundation of Chengdu Medical College (CYFY2019ZD06).

\section{ACKNOWLEDGMENTS}

This manuscript has been released as a pre-print at Research Square (50).

\section{SUPPLEMENTARY MATERIAL}

The Supplementary Material for this article can be found online at: https://www.frontiersin.org/articles/10.3389/fimmu.2020.593368/ full\#supplementary-material

clinical signs of experimental autoimmune encephalomyelitis. Int Immunopharmacol (2008) 8:1291-7. doi: 10.1016/j.intimp.2008.05.002

3. Ascherio A, Munger KL. Environmental risk factors for multiple sclerosis. Part I: the role of infection. Ann Neurol (2007) 61:288-99. doi: 10.1002/ana.21117

4. O'Gorman C, Lin R, Stankovich J, Broadley SA. Modelling genetic susceptibility to multiple sclerosis with family data. Neuroepidemiology (2013) 40:1-12. doi: 10.1159/000341902 
5. Liu F, Li Z, He X, Yu H, Feng J. Ghrelin attenuates neuroinflammation and demyelination in experimental autoimmune encephalomyelitis involving NLRP3 inflammasome signaling pathway and pyroptosis. Front Pharmacol (2019) 10:1320. doi: 10.3389/fphar.2019.01320

6. Correale J, Gaitán MI, Ysrraelit MC, Fiol MP. Progressive multiple sclerosis: from pathogenic mechanisms to treatment. Brain (2017) 140:527-46. doi: 10.1093/brain/aww258

7. Zhang D, Qiao L, Fu T. Paricalcitol improves experimental autoimmune encephalomyelitis (EAE) by suppressing inflammation via NF- $\mathrm{KB}$ signaling. BioMed Pharmacother (2020) 125:109528. doi: 10.1016/j.biopha.2019.109528

8. Pejman S, Kamarehei M, Riazi G, Pooyan S, Balalaie S. Ac-SDKP ameliorates the progression of experimental autoimmune encephalomyelitis via inhibition of ER stress and oxidative stress in the hippocampus of C57BL/6 mice. Brain Res Bull (2020) 154:21-31. doi: 10.1016/j.brainresbull.2019.09.014

9. Chen W, Zhao M, Zhao S, Lu Q, Ni L, Zou C, et al. Activation of the TXNIP/ NLRP3 inflammasome pathway contributes to inflammation in diabetic retinopathy: a novel inhibitory effect of minocycline. Inflamm Res (2017) 66:157-66. doi: 10.1007/s00011-016-1002-6

10. Hui ST, Andres AM, Miller AK, Spann NJ, Potter DW, Post NM, et al. Txnip balances metabolic and growth signaling via PTEN disulfide reduction. Proc Natl Acad Sci U S A (2008) 105:3921-6. doi: 10.1073/pnas.0800293105

11. Hou Y, Wang Y, He Q, Li L, Xie H, Zhao Y, et al. Nrf2 inhibits NLRP3 inflammasome activation through regulating Trx1/TXNIP complex in cerebral ischemia reperfusion injury. Behav Brain Res (2018) 336:32-9. doi: 10.1016/j.bbr.2017.06.027

12. Wree A, Eguchi A, McGeough MD, Pena CA, Johnson CD, Canbay A, et al. NLRP3 inflammasome activation results in hepatocyte pyroptosis, liver inflammation, and fibrosis in mice. Hepatology (2014) 59:898-910. doi: 10.1002/hep.26592

13. Chen X, He WT, Hu L, Li J, Fang Y, Wang X, et al. Pyroptosis is driven by non-selective gasdermin-D pore and its morphology is different from MLKL channel-mediated necroptosis. Cell Res (2016) 26:1007-20. doi: 10.1038/ cr.2016.100

14. Somacal S, Figueiredo CG, Quatrin A, Ruviaro AR, Conte L, Augusti PR, et al. The antiatherogenic effect of bixin in hypercholesterolemic rabbits is associated to the improvement of lipid profile and to its antioxidant and anti-inflammatory effects. Mol Cell Biochem (2015) 403:243-53. doi: 10.1007/ s11010-015-2354-X

15. Tao S, Rojo de la Vega M, Quijada H, Wondrak GT, Wang T, Garcia JG, et al. Bixin protects mice against ventilation-induced lung injury in an NRF2dependent manner. Sci Rep (2016) 6:18760. doi: 10.1038/srep18760

16. Tao S, Lakey-Beitia J, Kumar DJ, Hegde ML, Rao KS. Carotenoids as novel therapeutic molecules against neurodegenerative disorders: Chemistry and molecular docking analysis. Int J Mol Sci (2019) 20:e5553. doi: 10.3390/ ijms 20225553

17. de Oliveira Júnior RG, Bonnet A, Braconnier E, Groult H, Prunier G, Beaugeard L, et al. Bixin, an apocarotenoid isolated from Bixa orellana L., sensitizes human melanoma cells to dacarbazine-induced apoptosis through ROS-mediated cytotoxicity. Food Chem Toxicol (2019) 125:549-61. doi: $10.1016 /$ j.fct.2019.02.013

18. Rivera-Madrid R, Aguilar-Espinosa M, Cárdenas-Conejo Y, Garza-Caligaris LE. Carotenoid derivates in achiote (Bixa orellana) seeds: Synthesis and health promoting properties. Front Plant Sci (2016) 7:1406. doi: 10.3389/ fpls.2016.01406

19. $\mathrm{Xu} Z$, Kong XQ. Bixin ameliorates high fat diet-induced cardiac injury in mice through inflammation and oxidative stress suppression. BioMed Pharmacother (2017) 89:991-1004. doi: 10.1016/j.biopha.2017.02.052

20. Zhang H, Xue L, Li B, Tian H, Zhang Z, Tao S. Therapeutic potential of bixin in PM2.5 particles-induced lung injury in an Nrf2-dependent manner. Free Rad Biol Med (2018) 126:166-76. doi: 10.1016/j.freeradbiomed. 2018.08.015

21. Lu K, Liu L, Xu X, Zhao F, Deng J, Tang X, et al. ADAMTS13 ameliorates inflammatory responses in experimental autoimmune encephalomyelitis. J Neuroinflamm (2020) 17:67. doi: 10.1186/s12974-020-1713-Z

22. Liu $\mathrm{X}$, Zhu Q, Zhang $\mathrm{M}$, Yin $\mathrm{T}, \mathrm{Xu} \mathrm{R}$, Xiao $\mathrm{W}$, et al. Isoliquiritigenin ameliorates acute pancreatitis in mice via inhibition of oxidative stress and modulation of the Nrf2/HO-1 pathway. Oxid Med Cell Longev (2018) 2018:7161592. doi: 10.1155/2018/7161592
23. Qiu X, Guo Q, Liu X, Luo H, Fan D, Deng Y, et al. Pien Tze Huang alleviates relapsing-remitting experimental autoimmune encephalomyelitis mice by regulating Th1 and Th17 cells. Front Pharmacol (2018) 9:1237. doi: 10. 3389/fphar.2018.01237

24. Jiang HR, Milovanović M, Allan D, Niedbala W, Besnard AG, Fukada SY, et al. IL-33 attenuates EAE by suppressing IL-17 and IFN- $\gamma$ production and inducing alternatively activated macrophage. Eur J Immunol (2012) 42:180414. doi: 10.1002/eji.201141947

25. Scheiblich H, Trombly M, Ramirez A, Heneka MT. Neuroimmune connections in aging and neurodegenerative diseases. Trends Immunol (2020) 41:300-12. doi: 10.1016/j.it.2020.02.002

26. Wang D, Zhu B, Liu X, Han Q, Ge W, Zhang W, et al. Daphnetin Ameliorates Experimental Autoimmune Encephalomyelitis Through Regulating Heme Oxygenase-1. Neurochem Res (2020) 45:872-81. doi: 10.1007/s11064-020-02960-0

27. Tarantini S, Valcarcel-Ares MN, Yabluchanskiy A, Tucsek Z, Hertelendy P, Kiss T, et al. Nrf2 deficiency exacerbates obesity-induced oxidative stress, neurovascular dysfunction, blood-brain barrier disruption, neuroinflammation, amyloidogenic gene expression, and cognitive decline in mice, mimicking the aging phenotype. J Gerontol A Biol Sci Med Sci (2018) 73:853-63. doi: 10.1093/gerona/glx177

28. Milo R. Therapeutic strategies targeting B-cells in multiple sclerosis. Autoimmun Rev (2016) 15:714-8. doi: 10.1016/j.autrev.2016.03.006

29. Dang C, Han B, Li Q, Han R, Hao J. Up-regulation of PGC-1alpha in neurons protects against experimental autoimmune encephalomyelitis. FASEB J (2019) 33:14811-24. doi: 10.1096/fj.201901149RR

30. Kumar Y, Phaniendra A, Periyasamy L. Bixin triggers apoptosis of human Hep3B hepatocellular carcinoma cells: An insight to molecular and IN SILICO approach. Nutr Can (2018) 70:971-83. doi: 10.1080/01635581. 2018.1490445

31. Villacampa N, Heneka MT. Microglia in Alzheimer's disease: Local heroes! J Exp Med (2020) 217:e20192311. doi: 10.1084/jem.20192311

32. Khan N, Kuo A, Brockman DA, Cooper MA, Smith MT. Pharmacological inhibition of the NLRP3 inflammasome as a potential target for multiple sclerosis induced central neuropathic pain. Inflammopharmacology (2018) 26:77-86. doi: 10.1007/s10787-017-0401-9

33. Zsila F, Molnár P, Deli J, Lockwood SF. Circular dichroism and absorption spectroscopic data reveal binding of the natural cis-carotenoid bixin to human alpha1-acid glycoprotein. Bioorg Chem (2005) 33:298-309. doi: 10.1016/ j.bioorg.2005.03.003

34. Guo C, Fulp JW, Jiang Y, Li X, Chojnacki JE, Wu J, et al. Development and characterization of a hydroxyl-sulfonamide analogue, 5-chloro-N-[2-(4hydroxysulfamoyl-phenyl)-ethyl]-2-methoxy-benzamide, as a novel NLRP3 inflammasome inhibitor for potential treatment of multiple sclerosis. ACS Chem Neurosci (2017) 8:2194-201. doi: 10.1021/acschemneuro.7b00124

35. Anwar S, Rivest S. Alzheimer's disease: microglia targets and their modulation to promote amyloid phagocytosis and mitigate neuroinflammation. Expert Opin Ther Targets (2020) 24:331-44. doi: 10. 1080/14728222.2020.1738391

36. An K, Xue MJ, Zhong JY, Yu SN, Lan TS, Qi ZQ, et al. Arsenic trioxide ameliorates experimental autoimmune encephalomyelitis in C57BL/6 mice by inducing CD4 T cell apoptosis. J Neuroinflamm (2020) 17:147. doi: 10.1186/ s12974-020-01829-x

37. Braga TT, Brandao WN, Azevedo H, Terra FF, Melo ACL, Pereira FV, et al. NLRP3 gain-of-function in CD4+ T lymphocytes ameliorates experimental autoimmune encephalomyelitis. Clin Sci (Lond) (2019) 133:1901-16. doi: 10.1042/CS20190506

38. Soares JL, Oliveira EM, Pontillo A. Variants in NLRP3 and NLRC4 inflammasome associate with susceptibility and severity of multiple sclerosis. Mult Scler Relat Disord (2019) 29:26-34. doi: 10.1016/j.msard.2019.01.023

39. Lu L, Lu Q, Chen W, Li J, Li C, Zheng Z. Vitamin D3 protects against diabetic retinopathy by inhibiting high-glucose-induced activation of the ROS/ TXNIP/NLRP3 inflammasome pathway. J Diabetes Res (2018) 2018: 8193523. doi: 10.1155/2018/8193523

40. Inoue M, Shinohara ML. NLRP3 Inflammasome and MS/EAE. Autoimmune Dis (2013) 2013:859145. doi: 10.1155/2013/859145

41. Gris D, Ye Z, Iocca HA, Wen H, Craven RR, Gris P, et al. NLRP3 plays a critical role in the development of experimental autoimmune encephalomyelitis by mediating Th1 and Th17 responses. J Immunol (2010) 185:974-81. doi: 10.4049/ jimmunol.0904145 
42. Sánchez-Fernández A, Skouras DB, Dinarello CA, López-Vales R. OLT1177 (Dapansutrile), a Selective NLRP3 Inflammasome Inhibitor, Ameliorates Experimental Autoimmune Encephalomyelitis Pathogenesis. Front Immunol (2019) 10:2578. doi: 10.3389/fimmu.2019.02578

43. Han Y, Xu X, Tang C, Gao P, Chen X, Xiong X, et al. Reactive oxygen species promote tubular injury in diabetic nephropathy: The role of the mitochondrial ros-txnip-nlrp3 biological axis. Redox Biol (2018) 16:32-46. doi: 10.1016/ j.redox.2018.02.013

44. Wang DS, Yan LY, Yang DZ, Lyu Y, Fang LH, Wang SB, et al. Formononetin ameliorates myocardial ischemia/reperfusion injury in rats by suppressing the ROS-TXNIP-NLRP3 pathway. Biochem Biophys Res Commun (2020) 525:759-66. doi: 10.1016/j.bbrc.2020.02.147

45. Lian D, Dai L, Xie Z, Zhou X, Liu X, Zhang Y, et al. Periodontal ligament fibroblasts migration injury via ROS/TXNIP/Nlrp3 inflammasome pathway with Porphyromonas gingivalis lipopolysaccharide. Mol Immunol (2018) 103:209-19. doi: 10.1016/j.molimm.2018.10.001

46. Tan Y, Ichikawa T, Li J, Si Q, Yang H, Chen X, et al. Diabetic downregulation of Nrf2 activity via ERK contributes to oxidative stress-induced insulin resistance in cardiac cells in vitro and in vivo. Diabetes (2011) 60:625-33. doi: 10.2337/db10-1164

47. Murakami S, Motohashi H. Roles of Nrf2 in cell proliferation and differentiation. Free Rad Biol Med (2015) 88:168-78. doi: 10.1016/ j.freeradbiomed.2015.06.030
48. Rojo de la Vega M, Zhang DD, Wondrak GT. Topical bixin confers NRF2dependent protection against photodamage and hair graying in mouse skin. Front Pharmacol (2018) 9:287. doi: 10.3389/fphar.2018.00287

49. Xue L, Zhang H, Zhang J, Li B, Zhang Z, Tao S. Bixin protects against particleinduced long-term lung injury in an NRF2-dependent manner. Toxicol Res (Camb) (2018) 7:258-70. doi: 10.1039/c7tx00304h

50. Yu Y, Wu DM, Li J, Deng SH, Liu T, Zhang T, et al. Bixin attenuates experimental autoimmune encephalomyelitis by suppressing TXNIP/NLRP3 inflammasome activity and activating NRF2 signaling. Res Square (2020) doi: $10.21203 /$ rs.3.rs-24795/v1

Conflict of Interest: The authors declare that the research was conducted in the absence of any commercial or financial relationships that could be construed as a potential conflict of interest.

Copyright (c) $2020 \mathrm{Yu}, \mathrm{Wu}, \mathrm{Li}$, Deng, Liu, Zhang, He, Zhao and Xu. This is an open-access article distributed under the terms of the Creative Commons Attribution License (CC BY). The use, distribution or reproduction in other forums is permitted, provided the original author(s) and the copyright owner(s) are credited and that the original publication in this journal is cited, in accordance with accepted academic practice. No use, distribution or reproduction is permitted which does not comply with these terms. 